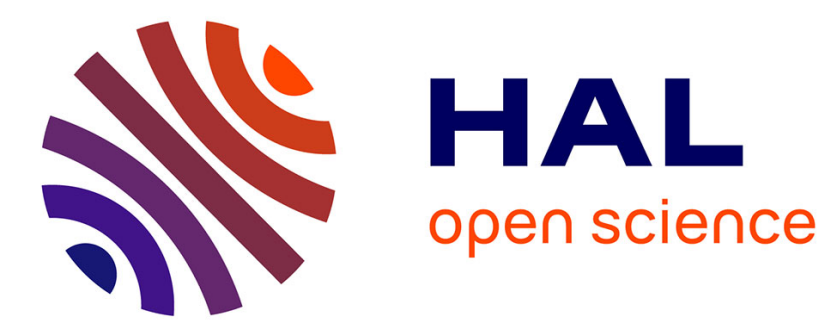

\title{
Piecewise-linear Models of Genetic Regulatory Networks: Equilibria and their Stability
}

\author{
Richard Casey, Hidde de Jong, Jean-Luc Gouzé
}

\section{To cite this version:}

Richard Casey, Hidde de Jong, Jean-Luc Gouzé. Piecewise-linear Models of Genetic Regulatory Networks: Equilibria and their Stability. [Research Report] RR-5353, INRIA. 2004. inria-00071250

\section{HAL Id: inria-00071250 \\ https://hal.inria.fr/inria-00071250}

Submitted on 23 May 2006

HAL is a multi-disciplinary open access archive for the deposit and dissemination of scientific research documents, whether they are published or not. The documents may come from teaching and research institutions in France or abroad, or from public or private research centers.
L'archive ouverte pluridisciplinaire HAL, est destinée au dépôt et à la diffusion de documents scientifiques de niveau recherche, publiés ou non, émanant des établissements d'enseignement et de recherche français ou étrangers, des laboratoires publics ou privés. 
INSTITUT NATIONAL DE RECHERCHE EN INFORMATIQUE ET EN AUTOMATIQUE

\title{
Piecewise-linear Models of Genetic Regulatory Networks: Equilibria and their Stability
}

\author{
Richard Casey — Hidde de Jong — Jean-Luc Gouzé
}

\section{$\mathbf{N}^{\circ} 5353$}

25 octobre 2004

Thème BIO

\section{apport}

de recherche 



\title{
Piecewise-linear Models of Genetic Regulatory Networks: Equilibria and their Stability
}

\author{
Richard Casey , Hidde de Jong * , Jean-Luc Gouzé \\ Thème BIO - Systèmes biologiques \\ Projets COMORE, HELIX \\ Rapport de recherche $n^{\circ} 5353-25$ octobre $2004-34$ pages
}

\begin{abstract}
A formalism based on piecewise-linear (PL) differential equations, originally due to Glass and Kauffman, has been shown to be well-suited to modelling genetic regulatory networks. However, the discontinuous vector field inherent in the PL models raises some mathematical problems in defining solutions on the surfaces of discontinuity. To overcome these difficulties we use the approach of Filippov, which extends the vector field to a differential inclusion. We study the stability of equilibria (called singular equilibrium sets) that lie on the surfaces of discontinuity. We prove several theorems that characterize the stability of these singular equilibria directly from the state transition graph, which is a qualitative representation of the dynamics of the system. We also formulate a stronger conjecture on the stability of these singular equilibrium sets.
\end{abstract}

Key-words: Piecewise-linear systems, Filippov solutions, stability, genetic regulatory networks

* INRIA Rhône-Alpes 


\section{Modèles linéaires par morceaux de réseaux de régulation génique : les points d'équilibre et leur stabilité}

Résumé : Nous utilisons pour modéliser les réseaux de régulation génétique un formalisme à base d'équations différentielles linéaires par morceaux, dû à Glass et Kauffman. Nous utilisons l'approche par inclusion différentielle due à Filippov pour définir les solutions sur les surfaces de discontinuité (les seuils). Nous étudions alors la stabilité des équilibres singuliers sur ces surfaces. Nous prouvons plusieurs théorèmes, qui permettent de déduire la stabilité asymptotique à partir du diagramme de transition, qui représente qualitativement les transitions possibles entre les différents domaines de l'espace des états.

Mots-clés : Systèmes linéaires par morceaux, solutions de Filippov, stabilité, réseaux géniques 


\section{Contents}

1 Introduction $\quad 4$

2 Piecewise-linear Models of Genetic Regulatory Networks 6

2.1 Domains in Phase Space . . . . . . . . . . . . . . . . . . 7

2.2 Classical Solutions and Focal Points . . . . . . . . . . . . . . . . . . 9

2.3 Filippov Solutions and Focal Sets . . . . . . . . . . . . . 10

3 Equilibria and Stability $\quad 13$

3.1 Regular Equilibrium Points . . . . . . . . . . . . . . . . . 14

3.2 Singular Equilibrium Points and Sets . . . . . . . . . . . . . . 14

4 Graphical Representation of the Dynamics $\quad 17$

4.1 States and Transitions . . . . . . . . . . . . . . . . . 17

4.2 State Transition Graph . . . . . . . . . . . . . . . . . . 20

5 Criteria for Stability of Singular Equilibria $\quad 21$

5.1 Theorem on Stability . . . . . . . . . . . . . . . . . . 21

5.2 Theorem on Instability . . . . . . . . . . . . . . . . 26

5.3 Stronger Conjecture on Stability . . . . . . . . . . . . . . 27

6 Discussion $\quad 29$

7 Acknowledgments $\quad 31$ 
Casey et al

\section{Introduction}

Given the central role the genome plays in the control of cellular processes, to fully understand the molecular basis for the functioning of living organisms, we need to determine which genes are expressed, when and where in an organism, and to what extent. The regulation of gene expression occurs through networks of regulatory interactions between DNA, RNA, proteins and small molecules, so-called genetic regulatory networks. Most networks of interest consist of many individual components interacting through complex positive and negative feedback loops, so the resulting behaviour is difficult to understand. This complexity means that, in addition to recent advances in experimental techniques in (post)genomics, mathematical modelling and computational techniques will be essential to the understanding of genetic regulatory networks (see [dJ02] for a review). The principal modelling challenges come from incomplete knowledge of the biochemical reactions underpinning most networks, and the dearth of quantitative data for kinetic parameters required for detailed mathematical models. Qualitative methods overcome both of these difficulties and are thus well-suited to the modelling and simulation of genetic regulatory networks.

A class of piecewise-linear (PL) models, originally proposed by Glass and Kauffman [GK73], is well suited to qualitative analysis and has been widely used in modelling genetic regulatory networks. The properties of these PL models have been well-studied in the mathematical biology literature, by for example Glass and Pasternack [GP78], Snoussi [Sno89], Plahte et al [PMO94], Mestl et al [MPO95b], Thomas et al [TTK95], Edwards [Edw00], Gouzé and Sari [GS02], and more recently in the hybrid systems literature by Ghosh and Tomlin [GT01], Alur and Belta $\left[\mathrm{ABI}^{+} 01\right]$, and Belta et al $\left[\mathrm{BFH}^{+} 04\right]$. The variables in the piecewise-linear differential equation (PLDE) models are the concentrations of proteins encoded by the genes, while the differential equations describe the regulatory interactions in the network by means of step functions. The use of step functions is motivated by the switch-like behaviour of many of the interactions in genetic regulatory networks [YY71, Pta92], but it does lead to some mathematical difficulties. The vector field for the PLDE model is undefined when one of the variables assumes a value where the step function is discontinuous, referred to as a threshold value. Recent work by Gouzé and Sari [GS02] uses an approach due to Filippov [Fil88] to define the solutions on the threshold hyperplanes. Widely used in control theory, the approach involves extending the PLDE to a piecewiselinear differential inclusion (PLDI). The solutions of the PLDI on the threshold hyperplanes are called sliding modes in the control literature. A qualitative simulation method based on PLDI models has been implemented in the software tool Genetic Network Analyzer (GNA) [dJGHP03], and has been used to analyze several regulatory networks of biological interest $\left[\mathrm{VF} 04, \mathrm{dJGB}^{+} 04, \mathrm{RdJP}^{+}\right]$. The qualitative simulation method used by GNA is based on the computation of a state transition graph, an abstraction of the qualitative dynamics of the PL system.

In order to analyze the dynamics of the system in phase space, we partition the phase space into domains bounded by the threshold hyperplanes. Then the phase space for the PL system is composed of domains where no variable takes a threshold value, referred to as regulatory domains, and the threshold hyperplanes and their intersections, where at least one

INRIA 
variable has a threshold value, referred to as switching domains. In addition to clarifying the definition of the vector field and its solutions in the switching domains, the PLDI approach enables the definition and computation of all equilibria for the PL system, including those that lie in switching domains.

Equilibria of the PL systems that lie in regulatory domains (so-called regular equilibria) are known to be asymptotically stable. Several authors have also studied the stability of periodic orbits in a restricted form of these PL systems [GP78, Edw00]. On the other hand, the characterization of attractors in switching domains (such as so-called singular equilibria) is complicated by two facts: the equilibria are not isolated points and are in general set-valued, and the usual uniqueness properties from the theory of ordinary differential equations do not apply to the PLDI models. The stability of equilibria for PL systems is an important subject in hybrid systems and control theory. See, for example, the review of Decarlo et al [DBPL00]. Despite some recent work on the stability of sliding mode solutions [JR98, GP01, LN04], and some classical papers [PS87, SP94], we found little in the literature that was practicable for studying stability of singular equilibria in this particular PLDI system. Most results make assumptions that are appropriate for large classes of control problems but do not hold for this system, such as the linear system having a common equilibrium point in all domains, or do not consider sliding modes [Bra98]. However, the structure of our particular PL system is such that a more tailored approach is feasible.

The study of the stability of equilibria in switching domains (singular equilibria) for PL models of genetic regulatory networks is the focus of this paper. There are two main contributions we present. Firstly, we extend the work of Gouzé and Sari [GS02] and de Jong et al $\left[\mathrm{dJGH}^{+} 04\right]$, putting the problem of stability in this class of PL systems into the framework of differential inclusions and Filippov solutions. Secondly, we prove several results on the stability of singular equilibria based on properties of the state transition graph. Criteria for stability that are formulated from properties of the state transition graph are both natural and practical, since one goal of this work is to incorporate stability criteria into the qualitative simulation method implemented in GNA.

The paper is organized as follows. In Section 2 we specify the PL system in detail, define the domains and discuss the Filippov solutions of the PLDI. In Section 3 we define regular and singular equilibrium points and sets. In Section 4 we give an overview of the graphical description of the qualitative dynamics of the PL system, the state transition graph, consisting of the domains and the transitions between them. We also state and prove a proposition on transitions that will turn out to be useful later on. In Section 6 we state and prove some theorems on the stability of singular equilibrium sets, and state an additional stronger conjecture. In the final section we present our conclusions and the discussion.

$\mathrm{RR} \mathrm{n}^{\circ} 5353$ 


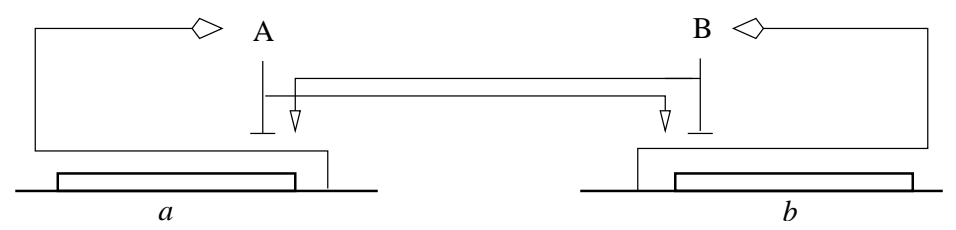

Figure 1: Example of a genetic regulatory network of two genes ( $a$ and $b$ ), each coding for a regulatory protein (A and $\mathrm{B})$. The notation follows in a simplified form the conventions of Kohn [Koh01].

\section{Piecewise-linear Models of Genetic Regulatory Net- works}

The schematic diagram in Figure 1 describes a simple genetic regulatory network. In this example, the genes $a$ and $b$ code for the proteins A and B, which in turn control the expression of the two genes $a$ and $b$. Protein A inhibits gene $a$ and activates gene $b$ above certain threshold concentrations, which are assumed to be different. Similarly protein B inhibits gene $b$ and activates gene $a$ above different threshold concentrations. This two-gene regulatory network is simple but represents many features of regulation found in real networks: auto-regulation, cross-regulation and inhibition/activation. Such a two-gene network could be found as a module of a more complex genetic regulatory network from a real biological system.

The dynamics of genetic regulatory networks can be modelled by a class of dynamical systems proposed originally by Glass and Kauffman [GK73]. The model has the general form

$$
\dot{x}_{i}=f_{i}(x)-\gamma_{i} x_{i}, \quad 1 \leq i \leq n,
$$

where $x=\left(x_{1}, \ldots, x_{n}\right)^{t}$ is a non-negative vector of protein concentrations. The non-negative quantities $f_{i}(x)$ and $\gamma_{i} x_{i}$ represent synthesis and degradation rates for each protein $x_{i}$ respectively. We can write the system (1) more compactly as

$$
\dot{x}=f(x)-\gamma x,
$$

where $f(x)=\left(f_{1}(x), \ldots, f_{n}(x)\right)^{t}$ and $\gamma=\operatorname{diag}\left(\gamma_{1}, \ldots, \gamma_{n}\right)$ is a constant diagonal matrix. The more general case when $\gamma=\gamma(x)$ is more complicated and will not be considered here. The functions $f_{i}: \mathbb{R}_{+}^{n} \rightarrow \mathbb{R}_{+}$represent the dependence of the rate of synthesis of a protein encoded by gene $i$ on the concentrations $x$ of protein in the cell. They can be written as

$$
f_{i}(x)=\sum_{i \in I} \kappa_{i l} b_{i l}(x),
$$

where $\kappa_{i l}>0$ is a rate parameter, $b_{i l}: \mathbb{R}_{+}^{n} \rightarrow\{0,1\}$ is a boolean-valued regulation function, and $I$ is an index set. The regulation functions $b_{i l}$ capture the conditions under which the 
protein encoded by gene $i$ is synthesized at a rate $\kappa_{i l}$. These conditions are written down as combinations of step functions $s^{+}, s^{-}: K \times \mathbb{R}_{+} \rightarrow\{0,1\}$, where

$$
s^{+}\left(x_{i}, \theta_{i}\right)=\left\{\begin{array}{ll}
1, & x_{i}>\theta_{i}, \\
0, & x_{i}<\theta_{i},
\end{array} \quad \text { and } \quad s^{-}\left(x_{i}, \theta_{i}\right)=1-s^{+}\left(x_{i}, \theta_{i}\right),\right.
$$

and $K \subseteq \mathbb{R}_{+}$. Here $x_{i}$ is a component of the concentration vector $x$ for the proteins, and the parameters $\theta_{i}$ are threshold concentrations. The use of such step functions has been motivated by the observation that the activity of a gene changes in a switch-like manner at a threshold concentration of a regulatory protein. The model (2) is piecewise-linear (PL) with the above definition of the $f_{i}$. Note that the step functions are not defined for $x_{i}=\theta_{i}$, so neither are the regulation functions. The parameters for the PL model are $\left\{\theta_{i}^{j}\right\},\left\{\gamma_{i}\right\},\left\{\kappa_{i l}\right\}$. The equations modelling the example network in Figure 1 can be written down as

$$
\begin{aligned}
& \dot{x}_{a}=\kappa_{a} s^{+}\left(x_{b}, \theta_{b}^{1}\right) s^{-}\left(x_{a}, \theta_{a}^{2}\right)-\gamma_{a} x_{a}, \\
& \dot{x}_{b}=\kappa_{b} s^{+}\left(x_{a}, \theta_{a}^{1}\right) s^{-}\left(x_{b}, \theta_{b}^{2}\right)-\gamma_{b} x_{b} .
\end{aligned}
$$

Gene $a$ is expressed at a rate $\kappa_{a}$ if the concentration $x_{b}$ of protein $\mathrm{B}$ is above the threshold $\theta_{b}^{1}$ and the concentration $x_{a}$ of protein $\mathrm{A}$ is below the threshold $\theta_{a}^{2}$. Similarly, gene $b$ is expressed at a rate $\kappa_{b}$ if the concentration $x_{a}$ of protein $\mathrm{A}$ is above the threshold $\theta_{a}^{1}$ and the concentration $x_{b}$ of the protein B is below the threshold $\theta_{b}^{2}$. Degradation of both proteins is assumed to be proportional to their own concentrations, so that the expression of the genes $a$ and $b$ is modulated by the degradation rates $\gamma_{a} x_{a}$ and $\gamma_{b} x_{b}$ respectively. When necessary, the following parameter values will be assumed: $\theta_{a}^{1}=4, \theta_{a}^{2}=8, \theta_{b}^{1}=4, \theta_{b}^{2}=8, \kappa_{a}=20$, $\kappa_{b}=20, \gamma_{a}=2$, and $\gamma_{b}=2$.

\subsection{Domains in Phase Space}

The dynamics of the piecewise-linear system (2) can be studied in the $n$-dimensional phase space $\Omega=\Omega_{1} \times \ldots \times \Omega_{n}$, where each $\Omega_{i}$ is defined by $\Omega_{i}=\left\{x_{i} \in \mathbb{R}_{+} \mid 0 \leq x_{i} \leq \max _{i}\right\}$, for some positive parameter $\max _{i}$, with $\max _{i}>\max _{x \in \Omega}\left(f_{i}(x) / \gamma_{i}\right)$. A protein encoded by a gene will be involved in different interactions at different concentration thresholds, so for each variable $x_{i}$ we assume there are $p_{i}$ ordered thresholds $\theta_{i}^{1}, \ldots, \theta_{i}^{p_{i}}$. The $(n-1)$-dimensional hyperplanes defined by these thresholds partition $\Omega$ into hyper-rectangular regions we call domains. Specifically, a domain $D \subset \Omega$ is defined to be a set $D=D_{1} \times \cdots \times D_{n}$, where 
each set $D_{i}$, for $1 \leq i \leq n$, is given by one of the following equations

$$
\begin{aligned}
D_{i} & =\left\{x_{i} \in \Omega_{i} \mid 0 \leq x_{i}<\theta_{i}^{1}\right\}, \\
D_{i} & =\left\{x_{i} \in \Omega_{i} \mid x_{i}=\theta_{i}^{1}\right\}, \\
D_{i} & =\left\{x_{i} \in \Omega_{i} \mid \theta_{i}^{1}<x_{i}<\theta_{i}^{2}\right\}, \\
D_{i} & =\left\{x_{i} \in \Omega_{i} \mid x_{i}=\theta_{i}^{2}\right\}, \\
& \vdots \\
D_{i} & =\left\{x_{i} \in \Omega_{i} \mid x_{i}=\theta_{i}^{p_{i}}\right\}, \\
D_{i} & =\left\{x_{i} \in \Omega_{i} \mid \theta_{i}^{p_{i}}<x_{i} \leq \max _{i}\right\} .
\end{aligned}
$$

Let $\mathcal{D}$ denote the set of all domains in $\Omega$. The total number of domains in $\Omega$ is $\prod_{i=1}^{n}\left(2 p_{i}+1\right)$. A domain $D \in \mathcal{D}$ is called a regulatory domain if none of the variables $x_{i}$ has a threshold value in $D$. In contrast, a domain $D \in \mathcal{D}$ is called a switching domain if at least one of the variables has a threshold value in $D$. The corresponding variables $x_{i}$ are called switching variables in $D$. For convenience, we denote the sets of regulatory and switching domains by $\mathcal{D}_{r}$ and $\mathcal{D}_{s}$ respectively. Following Mestl et al [MPO95a], we define the order of a switching domain to be the number of switching variables in that domain.

Definition 1. The order of a domain $D \in \mathcal{D}$ is the number $k \in \mathbb{N}, 0 \leq k \leq n$ equal to the number of switching variables in $D$, denoted $\operatorname{order}(D)$.

It is also useful to define the concept of a supporting hyperplane for a domain.

Definition 2. For every domain $D \in \mathcal{D}_{s}$ of order $k \geq 1$, define $\operatorname{supp}(D) \subset \Omega$ to be the $(n-k)$-dimensional hyperplane containing $D$. If $D \in D_{r}$ then we define $\operatorname{supp}(D)$ to be equal to $\Omega$.

For every domain $D$ we can define the boundary of $\mathrm{D}$ in $\operatorname{supp}(D)$ to be the set $\partial D$ of all points $x \in \operatorname{supp}(D)$, such that each ball $B(x, \varepsilon)$ with centre $x$ and radius $\varepsilon>0$ intersects both $D$ and $\operatorname{supp}(D) \backslash D$. Then for every $D \in \mathcal{D}$ we can define the useful sets

$$
\begin{aligned}
& A(D)=\left\{D^{\prime} \in \mathcal{D} \mid D^{\prime} \subseteq \partial D\right\}, \text { and } \\
& R(D)=\left\{D^{\prime} \in \mathcal{D}_{r} \mid D \subseteq \partial D^{\prime}\right\} .
\end{aligned}
$$

The set $A(D) \subset \mathcal{D}$ contains the domains in the boundary of $D$, which are always switching domains, whereas $R(D) \subset \mathcal{D}_{r}$ is the set of regulatory domains that have $D$ in their boundary. Two domains $D$ and $D^{\prime}$ will be said contiguous if $D \in A\left(D^{\prime}\right)$ or $D^{\prime} \in A(D)$.

Remark. We can also characterize every domain $D \in \mathcal{D}$ in another manner that will prove convenient later. Suppose $D$ has order $k \geq 1$. Let $I$ be a subset of $k$ elements of $\{1, \ldots, n\}$. Let $q:\{1, \ldots, n\} \rightarrow \mathbb{N}, i \mapsto q_{i}$ be a mapping that indexes the domains by the thresholds and satisfies $1 \leq q_{i} \leq p_{i}$ if $i \in I$, and $0 \leq q_{i} \leq p_{i}$ if $i \notin I$. Then the domain $D$ of order $k$, defined by $(I, q)$, is

$$
D=\left\{x \in \Omega \mid x_{i}=\theta_{i}^{q_{i}} \text { if } i \in I, \theta_{i}^{q_{i}}<x_{i}<\theta_{i}^{q_{i}+1} \text { if } i \notin I\right\},
$$


where we have the convention that $\theta_{i}^{0}=0$ and $\theta_{i}^{p_{i}+1}=\max _{i}$, and $\theta_{i}^{0} \leq x_{i}$ and $x_{i} \leq \theta_{i}^{p_{i}+1}$, if $i \notin I$, so as to include the bounds of the domain $\Omega$.

In Figure 2(a) the 2-dimensional phase space $\Omega$ for the example two-gene network is shown. The phase space $\Omega$ is partitioned into 9 regulatory domains and 16 switching domains, since the protein concentrations have two thresholds each. For example, the domain $D^{1}=\left\{\left(x_{a}, x_{b}\right) \in \Omega \mid 0 \leq x_{a}<\theta_{a}^{1}, 0 \leq x_{b}<\theta_{b}^{1}\right\}$, is a regulatory domain, while $D^{2}=\left\{\left(x_{a}, x_{b}\right) \in \Omega \mid 0 \leq x_{a}<\theta_{a}^{1}, x_{b}=\theta_{b}^{1}\right\}$ is a switching domain. The only switching variable in $D^{2}$ is $x_{b}$, so $D^{2}$ is a domain of order 1 . Here $\operatorname{supp}\left(D^{2}\right)=\left\{\left(x_{a}, x_{b}\right) \in \Omega \mid x_{b}=\theta_{b}^{1}\right\}$, for example.
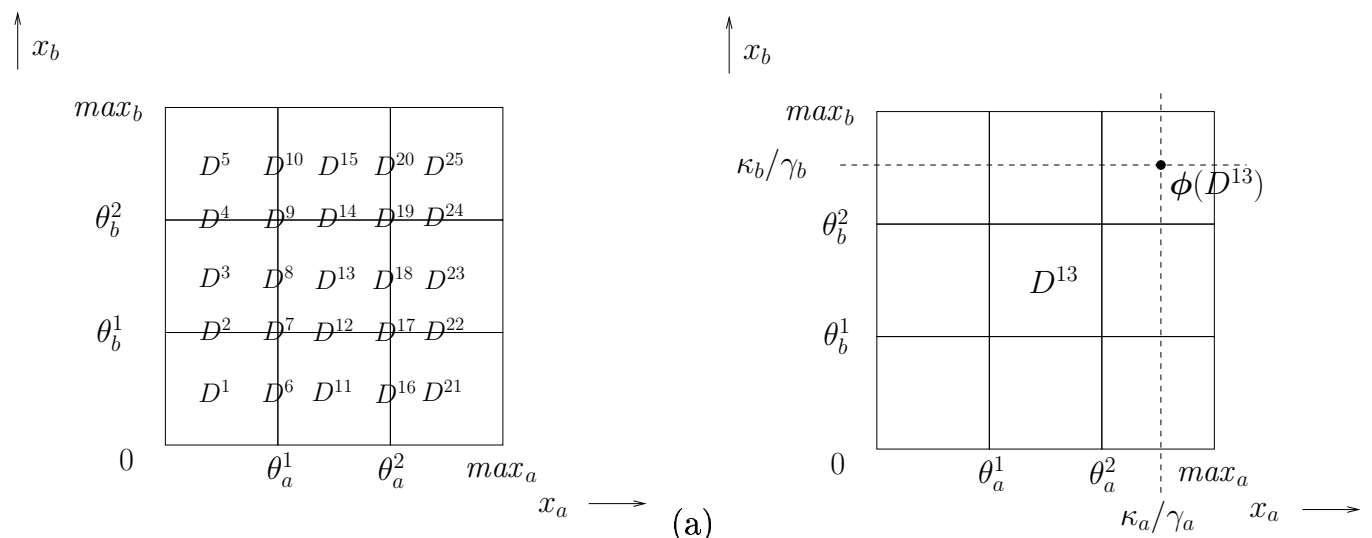

(a)

Figure 2: (a) Phase space box $\Omega$ for the PL model in Figure 1. (b) Focal point $\phi\left(D^{13}\right)$ for the regulatory domain $D^{13}$ : the variables $x_{a}$ and $x_{b}$ converge towards focal point values $\kappa_{a} / \gamma_{a}$ and $\kappa_{b} / \gamma_{b}$, respectively. With the parameter values from Section 2, the focal point lies in the domain $D^{25}$.

\subsection{Classical Solutions and Focal Points}

For any regulatory domain $D \in \mathcal{D}_{r}$, the function $f(x)$ is constant for all $x \in D$, and it follows that the piecewise-linear system (2) can be written as a linear vector field

$$
\dot{x}=f^{D}-\gamma x, \quad x \in D
$$

where $f^{D}$ is constant in $D$. Restricted to $D$, this is a classical linear ordinary differential equation. We assume that the parameters $\left\{\theta_{i}^{j}\right\},\left\{\gamma_{i}\right\},\left\{\kappa_{i l}\right\}$ are all fixed. For any initial condition $x\left(t_{0}\right) \in D$, the unique solution is given by

$$
x(t)=\phi(D)+e^{\gamma\left(t_{0}-t\right)}\left(x\left(t_{0}\right)-\phi(D)\right),
$$

where $\phi(D)$ satisfies the linear system $\gamma \phi(D)=f^{D}$. Clearly $x(t) \rightarrow \phi(D)$ monotonically as $t \rightarrow \infty$, until $x(t)$ reaches the boundary of the regulatory domain $D$.

$\mathrm{RR} \mathrm{n}^{\circ} 5353$ 
Definition 3. Given a regulatory domain $D \in \mathcal{D}_{r}$, the point $\phi(D)=\gamma^{-1} f^{D} \in \Omega$ is called the focal point for the flow in $D$.

Generally we make the assumption that $\phi(D) \notin \operatorname{supp}\left(D^{\prime}\right)$, for all $D^{\prime} \subseteq \partial D$, for otherwise solutions can take infinite time to reach a focal point in the boundary of their domain. This is a special case of a more general assumption we make in Section 2.3. In the example network of Figure 1, it can easily be checked that for the regulatory domain $D^{13}$, the state equations reduce to

$$
\begin{aligned}
\dot{x}_{a} & =\kappa_{a}-\gamma_{a} x_{a}, \\
\dot{x}_{b} & =\kappa_{b}-\gamma_{b} x_{b} .
\end{aligned}
$$

Hence the focal point of $D^{13}$ is $\phi\left(D^{13}\right)=\left(\kappa_{a} / \gamma_{a}, \kappa_{b} / \gamma_{b}\right)$, which lies outside $D^{13}$, in the domain $D^{25}$ in fact, as shown in Figure 2(b). Thus solutions in $D^{13}$ will flow towards $\phi\left(D^{13}\right) \in D^{25}$ until they leave the domain $D^{13}$. Different regulatory domains will usually have different focal points. In general, all solutions in a regulatory domain $D$ flow towards the focal point $\phi(D)$ until they either reach it or leave the domain $D$. What happens when a solution leaves a regulatory domain $D$ and enters a switching domain in the boundary of $D$ ? Since the step functions are not defined when a variable $x_{i}$ takes some threshold value $\theta_{i}^{q_{i}}$, the vector field is undefined on the switching domains. This can lead to mathematical difficulties if trajectories in different regulatory domains flow into the same switching domain. To regularise the ambiguity in how to continue such solutions, we use an approach originally due to Filippov.

\subsection{Filippov Solutions and Focal Sets}

In switching domains, the PL system (2) is not defined, since in a switching domain of order $k \geq 1, k$ variables assume a threshold value. Sometimes the solution in a regulatory domain reaches a switching domain from which it can be continued into a contiguous regulatory domain without difficulties [Sno89, ESAG01]. Such switching domains have been called transparent walls [MPO95a, PMO94], e.g. the switching domain $D^{6}$ in the example network, shown in Figure 3. However, if the solutions in two regulatory domains flow towards the same switching domain, then mathematical difficulties can arise. For example, consider the solutions arriving at $D^{14}$ from $D^{13}$ and $D^{15}$ in Figure 3. Such switching domains have been called black walls in the literature.

In order to define the solutions on switching domains, we use a construction originally proposed by Filippov [Fil88] and recently applied to PL systems of this form [GS02, d $\left.\mathrm{JGH}^{+} 04\right]$. The Filippov approach is much used in control theory, where the solutions on switching domains are known as sliding modes [ES98, Utk92], and the approach is consistent with the behaviour of the solutions seen in numerical simulation of PL systems (e.g. using Euler's method or another numerical integration scheme). The method consists of extending the system (8) to a differential inclusion,

$$
\dot{x} \in H(x)
$$



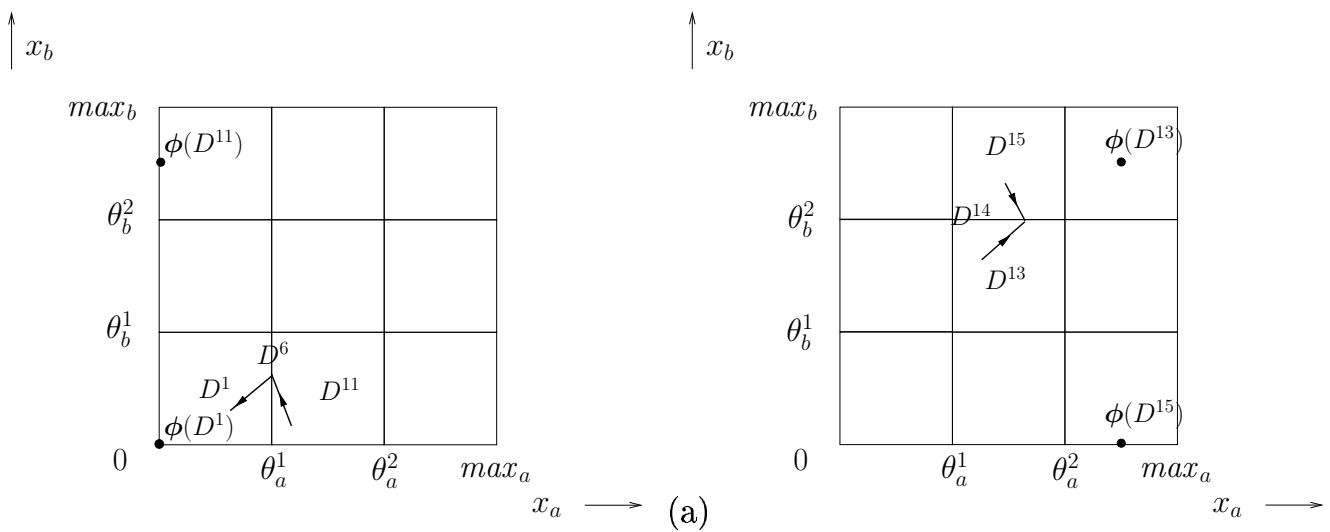

Figure 3: Behavior of the system of Figure 1 at some of the thresholds. The domains $D^{1}, D^{6}, D^{11}, D^{13}, D^{14}$ and $D^{15}$ are shown, in addition to the focal points $\phi\left(D^{1}\right)=(0,0)$, $\phi\left(D^{11}\right)=\left(0, \kappa_{b} / \gamma_{b}\right), \phi\left(D^{13}\right)=\left(\kappa_{a} / \gamma_{a}, \kappa_{b} / \gamma_{b}\right)$ and $\phi\left(D^{15}\right)=\left(\kappa_{a} / \gamma_{a}, 0\right)$. (a) The solution trajectories in $D^{11}$ can be continued in $D^{1}$, so $D^{6}$ is a transparent wall, whereas in (b), the solution trajectories in $D^{13}$ cannot be continued in $D^{15}$. The behavior of the system in $D^{14}$ cannot be determined without a Filippov-like approach.

where $H: \Omega \rightarrow 2^{\mathbb{R}^{n}}$ is a set-valued function. If $D$ is a regulatory domain, then we define $H$ simply as

$$
H(x)=\left\{f^{D}-\gamma x\right\},
$$

for $x \in D$. If $D$ is a switching domain, for $x \in D$, we define $H(x)$ as

$$
H(x)=\overline{c o}\left(\left\{f^{D^{\prime}}-\gamma x \mid D^{\prime} \in R(D)\right\}\right),
$$

where we recall $R(D)=\left\{D^{\prime} \in \mathcal{D}_{r} \mid D \subseteq \partial D^{\prime}\right\}$ is the set of all regulatory domains with $\mathrm{D}$ in their boundary, and $\overline{c o}(X)$ is the closed convex hull of $X$. For switching domains, $H(x)$ is generally multi-valued so we define solutions of the differential inclusion as follows.

Definition 4. An absolutely continuous function $\xi_{t}\left(x_{0}\right)$ is a solution of $(10)$ on $[0, T]$ in the sense of Filippov if $\xi_{0}\left(x_{0}\right)=x_{0}$ and $\dot{\xi}_{t} \in H\left(\xi_{t}\right)$, for almost all $t \in[0, T]$.

Indeed, for a solution $\xi_{t}$ in the sense of Filippov, we have that $\dot{\xi}_{\tau}$ is undefined at the instant $\tau \in[0, T]$ when $\xi_{t}$ reaches or leaves a threshold plane. Hereafter we will usually refer to "solutions in the sense of Filippov" as "Filippov solutions" or simply as "solutions" when discussing solutions of the differential inclusion $\dot{x} \in H(x)$.

It is useful to define a concept analogous to the focal points defined for regulatory domains, extended to deal with switching domains.

Definition 5. Let $D \in \mathcal{D}$ be a domain. If $D$ is a regulatory domain then its focal set $\Phi(D)$ is given by

$$
\Phi(D)=\{\phi(D)\},
$$


where $\phi(D)$ is the focal point of $D \in \mathcal{D}_{r}$ as in Definition 3. If $D$ is a switching domain of order $k$, and $\operatorname{supp}(D)$ is the $(n-k)$-dimensional hyperplane supporting $D$, then its focal set $\Phi(D)$ is

$$
\Phi(D)=\operatorname{supp}(D) \cap \overline{c o}\left(\left\{\phi\left(D^{\prime}\right) \mid D^{\prime} \in R(D)\right\}\right) .
$$

Hence $\Phi(D)$ for $D \in \mathcal{D}_{s}$ is the convex hull of the focal points $\phi\left(D^{\prime}\right)$ of all the regulatory domains $D^{\prime}$ having $D$ in their boundary, as defined above, intersected with the threshold hyperplane $\operatorname{supp}(D)$ containing the switching domain $D$. Thus $\Phi(D)$ can be a singleton, but more generally is a closed convex bounded set and hence is referred to as a focal set. Gouzé and Sari have shown that all solutions either cross a switching domain instantaneously, or remain in it while converging towards $\Phi(D)$, in the case when $\Phi(D)$ is a point [GS02]. The solutions that remain in a switching domain are called sliding modes. These sliding modes can occur in $D$ when

$$
\Phi(D) \neq\{\} .
$$

Further, for $D \in \mathcal{D}_{s}$, if $\Phi(D) \neq\{\}$ and $\Phi(D) \cap D=\{\}$, then all solutions will eventually leave the switching domain. If $\Phi(D) \neq\{\}$ and $\Phi(D) \cap D \neq\{\}$, then there are points in $D$ that behave like a set of equilibrium points in the classical sense. Under what conditions a set $\Phi(D)$ with $\Phi(D) \cap D \neq\{\}$ will be stable in some sense is the subject of the remainder of the paper ${ }^{1}$.

For a switching domain $D$ with $\Phi(D) \neq\{\}$, all solutions converge towards $\Phi(D)$ in a restricted sense. The convergence is monotonic in the sense that for all components $\left(\xi_{t}\right)_{i}$ of Filippov solutions $\xi_{t}(x)$ in $D$, each $\left(\xi_{t}\right)_{i}$ monotonically converges towards the projection of $\Phi(D)$ onto $\Omega_{i}$. This monotonic convergence property is summarized in the following lemma, which is also proven in the appendix of de Jong et al [dJGH $\left.{ }^{+} 04\right]$.

Lemma 2.1. For every regulatory domain $D \in \mathcal{D}_{r}$, all solutions $\xi_{t}$ in $D$ monotonically converge towards the focal set $\Phi(D)$. For every switching domain $D \in \mathcal{D}_{s},\left(\xi_{t}\right)_{i}$ monotonically converges towards the closed interval

$$
\pi_{i}(\Phi(D))=\left\{\phi_{i} \in \Omega_{i} \mid \phi \in \Phi(D)\right\},
$$

the projection of $\Phi(D)$ onto $\Omega_{i}$, if $\left(\xi_{0}\right)_{i} \notin \pi_{i}(\Phi(D))$. This holds for all components $\left(\xi_{t}\right)_{i}$ of $\xi_{t}, 1 \leq i \leq n$.

Proof: If $D$ is a regulatory domain then $\Phi(D)=\{\phi(D)\}$, and the solution $\xi_{t}$ in $D$ is given by the classical solution (9). It is clear from (9), that for any $x_{0} \in D \in \mathcal{D}_{r}, \xi_{t}\left(x_{0}\right) \rightarrow \phi(D)$ monotonically as $t \rightarrow \infty$, until $\xi_{t}\left(x_{0}\right)$ reaches $\partial D$. If $D$ is a switching domain then $\Phi(D)$ is set-valued. Assume initially that $\left(\xi_{t}\right)_{i}<\min \pi_{i}(\Phi(D))$ on $[0, \tau]$, with $\tau \geq 0$. From Definition 5 it follows that $\left(\xi_{t}\right)_{i}<\phi_{i}\left(D^{\prime}\right)=f_{i}^{D^{\prime}} / \gamma_{i}$ for all $D^{\prime} \in R(D)$. From (12), the differential inclusion on $D \in D_{s}$ is given by the set

$$
H\left(\xi_{t}\right)=\left\{\sum_{D^{\prime} \in R(D)} \alpha_{D^{\prime}}\left(f^{D^{\prime}}-\gamma \xi_{t}\right) \mid \sum_{D^{\prime} \in R(D)} \alpha_{D^{\prime}}=1, \alpha_{D^{\prime}} \geq 0\right\} .
$$

\footnotetext{
set.

${ }^{1}$ See also the treatment in de Jong et al $\left[\mathrm{dJGH}^{+} 04\right]$, where $\Phi(D)$ is referred to as a target equilibrium
} 
Since $\left.\dot{\xi}_{t} \in H\left(\xi_{t}\right)\right)$, we have that $\left(\dot{\xi}_{t}\right)_{i}>0$. Thus $\left(\xi_{t}\right)_{i}$ monotonically converges towards $\pi_{i}(\Phi(D))$ while it remains in $D$. The case $\left(\xi_{t}\right)_{i}>\max \pi_{i}(\Phi(D))$ is proven in a similar way.

The same proof also gives the following corollary. By a slight abuse of notation, we will denote $\Pi(D)$ the smallest closed hyperrectangle in $\operatorname{supp}(D)$ containing $\Phi(D)$, that is the hyperrectangle the projection of which on the $i$-th axe is $\pi_{i}(\Phi(D))$ for all $i$.

Corollary 1. All solutions $\xi_{t}$ in $D$ converge towards $\Pi(D)$, if $\xi_{0} \notin \Pi(D)$. $\Pi(D)$ is positively invariant.

Corollary 2. If $\Phi(D)$ is a point, all solutions $\xi_{t}$ in $D$ converge monotonically towards $\Phi(D)$.

The above lemma and corollaries will be used in several proofs later in the paper. To facilitate our discussion, we make a technical assumption on the focal sets for our system. The assumption rules out some more difficult cases when proving results on stability later in the paper.

Assumption 1. For all domains $D \in \mathcal{D}$,

$$
\Phi(D) \cap \operatorname{supp}\left(D^{\prime}\right)=\{\}, \forall D^{\prime} \subseteq \partial D .
$$

It essentially says that for every domain $D$, both switching and regulatory, the focal set $\Phi(D)$ does not intersect the supporting hyperplane of some domain $D^{\prime}$ in the boundary of $D$. For regulatory domains, Assumption 1 is equivalent to the assumption that $\phi(D) \notin \operatorname{supp}\left(D^{\prime}\right)$ for all $D^{\prime} \subseteq \partial D$. In fact, for regulatory domains this assumption is the generic case, but this is not true for switching domains. We will return to discuss the consequences of Assumption 1 in Section 6. The following corollary of the assumption will also be used in the following, and is easily deduced from the fact that all domains $D$ and their support are parallel to the axes, like the hyperrectangular region $\Pi(D)$.

Corollary 3. For all domains $D \in \mathcal{D}$,

$$
\Pi(D) \cap \operatorname{supp}\left(D^{\prime}\right)=\{\}, \forall D^{\prime} \subseteq \partial D .
$$

\section{Equilibria and Stability}

The focal sets discussed above are not, in general, true equilibria for the system as a whole, but under certain conditions they can be. If $D \in \mathcal{D}_{r}$ is a regulatory domain then $\Phi(D)$ is a singleton and if $\Phi(D) \in D$, it is known that $\Phi(D)$ is a regular equilibrium point for the system and is asymptotically stable. On the other hand, if $D \in \mathcal{D}_{s}$ is a switching domain then $\Phi(D)$ is generally a set and the situation is more complex. If $\Phi(D) \cap D \neq\{\}$ and Assumption 1 holds, then $\Phi(D)$ is a singular equilibrium set for the system and under certain conditions may be shown to be asymptotically stable in some appropriate sense. These concepts are explained more completely in the following sections.

$\mathrm{RR} \mathrm{n}^{\circ} 5353$ 


\subsection{Regular Equilibrium Points}

Recall that in a regulatory domain $D \in \mathcal{D}_{r}$, the differential inclusion reduces to the linear system

$$
\dot{x}=f^{D}-\gamma x,
$$

with $\gamma=\operatorname{diag}\left(\gamma_{1}, \ldots, \gamma_{n}\right)$, a constant positive diagonal matrix. The solutions are given by

$$
x(t)=\phi(D)+e^{\gamma\left(t_{0}-t\right)}\left(x\left(t_{0}\right)-\phi(D)\right),
$$

where $\phi(D)$ satisfies $\phi(D)=\gamma^{-1} f^{D}$. We have seen that $\phi(D) \in \Omega$ is the focal point of the flow in the regulatory domain $D$. Under Assumption 1 , if $\phi(D) \notin D$ then all solutions converge monotonically towards $\phi(D)$ until they leave the domain $D$. Alternatively, if $\phi(D) \in D$ then as $t \rightarrow \infty$ all solutions starting in $D$ converge towards $\phi(D)$, which is then a stable equilibrium point. We recall the following elementary result, originally due to Glass and Kauffman [GK73].

Theorem 3.1. Let $D$ be a regulatory domain with focal point $\phi(D)$. If $\phi(D) \in D$ then $x=\phi(D)$ is an asymptotically stable equilibrium point of (2).

Hence any focal points lying within their associated regulatory domain are always asymptotically stable, and we refer to them as regular equilibrium points. These points have also been called regular stationary points (RSPs) in the literature [ST93, MPO95a]. In the example network of Figure 1, it can be seen that for the regulatory domain $D^{1}$ the model reduces to

$$
\begin{aligned}
\dot{x}_{a} & =-\gamma_{a} x_{a}, \\
\dot{x}_{b} & =-\gamma_{b} x_{b} .
\end{aligned}
$$

Hence the focal point of $D^{1}$ is $\phi\left(D^{1}\right)=(0,0)$, which lies inside $D^{1}=\left\{\left(x_{a}, x_{b}\right) \in \Omega \mid 0 \leq\right.$ $\left.x_{a}<\theta_{a}^{1}, 0 \leq x_{b}<\theta_{b}^{1}\right\}$. Hence $(0,0)$ is a regular equilibrium point for the example network, and is asymptotically stable by Theorem 3.1 (see Figure 4). This stable regular equilibrium point represents a state of the network where both gene $a$ and gene $b$ are off.

\subsection{Singular Equilibrium Points and Sets}

Since regular equilibrium points are well studied and are always asymptotically stable, we focus on equilibrium points in switching domains for the remainder of the paper. In a switching domain $D \in \mathcal{D}_{s}$, recall that solutions are defined by considering the differential inclusion $H(x)$. We say that a point $y \in \Omega$ is an equilibrium point for the differential inclusion if

$$
0 \in H(y),
$$

where $H$ is computed using the Filippov construction as in (12). In other words, there is a solution in the sense of Filippov, $\xi_{t}$, such that $\xi_{t}(y)=y, \forall t>0$. We call such a point a singular equilibrium point, although they have also been called singular stationary points (SSPs) in the literature [ST93]. Given a switching domain $D \in \mathcal{D}_{s}$, we can check for singular 
$\uparrow x_{b}$

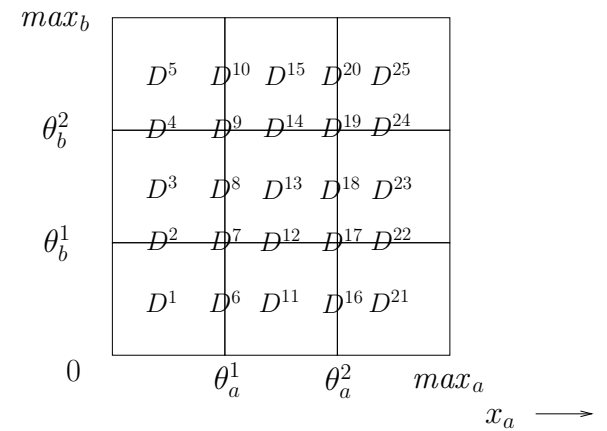

$x_{b}$

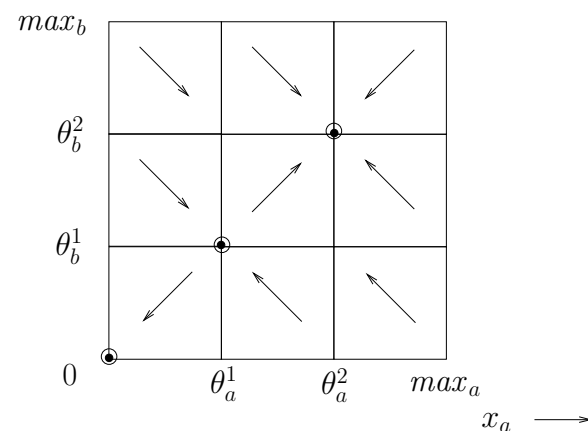

Figure 4: (a) Phase space box $\Omega$ for the PL model in Figure 1 showing all the domains. (b) Sketch of the vector field for the PL model in Figure 1, with the parameter values from Section 2. There is a regular equilibrium point at $(0,0) \in D^{1}$ and there are singular equilibrium points in $D^{7}$ and $D^{19}$.

equilibrium points by computing the focal set $\Phi(D)$. Under Assumption 1 , if $\Phi(D) \cap D \neq\{\}$ then we can deduce that $\Phi(D) \subseteq D$, i.e. that $D$ strictly contains or equals its focal set $(\Phi(D)$ being a closed set, equality only occurs if $D$ is a point). In fact, then every $\phi \in \Phi(D)$ is a singular equilibrium point of the differential inclusion. That is, for each $\phi \in \Phi(D)$, there exists a solution $\xi_{t}$ such that $\xi_{t}(\phi)=\phi, \forall t \geq 0$. Hence $\Phi(D)$ is a set of singular equilibrium points for the system, and we refer to such a $\Phi(D)$ as a singular equilibrium set.

In the example network of Figure 1, it can be seen from applying Definition 5 to the switching domain $D^{19}$ that the target equilibrium $\Phi\left(D^{19}\right)$ is given by $D^{19}$ itself. In this example, $D^{19}$ has order 2 in a 2 -dimensional phase space $\Omega$, so $D^{19}$ is simply a point and $\Phi\left(D^{19}\right)$ is a singleton. Hence $\Phi\left(D^{19}\right)$ is a singular equilibrium point and from examining the local behaviour of the vector field in Figure 4, it appears to be stable in some sense. Similarly, $\Phi\left(D^{7}\right)$ is a singular equilibrium point and from examining the local behaviour of the vector field, it appears to be a saddle-like point and hence unstable.

The fact that equilibria in switching domains can be set-valued, in addition to the nonuniqueness of solutions to differential inclusions, requires some extended definitions of stability. Filippov uses the term weakly stable to describe a equilibrium point that is stable in the sense of Lyapunov for some solution of the differential inclusion [Fil88]. The term stable is reserved for a stronger concept of stability, and describes a equilibrium point that is stable in the sense of Lyapunov for every solution of the differential inclusion. Asymptotically stable and weakly asymptotically stable can be defined similarly. These concepts of stable and weakly stable equilibrium points can be extended naturally to define stability for equilibrium sets. The definitions below are motivated by the standard definitions of 
stability for equilibrium points as found in Hirsch and Smale [HS74], and the formulation of Lyapunov stability for sets in Bhatia and Szegö [BS67].

Definition 6. An equilibrium set $E$ is stable if, for all neighbourhoods $V$ with $E \subseteq V$, there exists $U$ such that $E \subseteq U \subseteq V$ and for all $x \in U$, and for every solution $\xi_{t}$ of (10) with $\xi_{0}(x)=x$,

$$
\xi_{t}(x) \in V, \forall t \geq 0 .
$$

Definition 7. An equilibrium set $E$ is weakly stable if, for all neighbourhoods $V$ with $E \subseteq V$, there exists $U$ such that $E \subseteq U \subseteq V$ and for all $x \in U$, and for some solution $\xi_{t}$ of (10) with $\xi_{0}(x)=x$,

$$
\xi_{t}(x) \in V, \forall t \geq 0 .
$$

Definition 8. An equilibrium set $E$ is asymptotically stable if, for all neighbourhoods $V$ with $E \subseteq V$, there exists $U$ such that $E \subseteq U \subseteq V$ and for all $x \in U$, and for every solution $\xi_{t}$ of $(10)$ with $\xi_{0}(x)=x$,

1. $\xi_{t}(x) \in V, \forall t \geq 0$ and

2. $\lim _{t \rightarrow \infty} \xi_{t}(x) \in E$

Definition 9. An equilibrium set $E$ is weakly asymptotically stable if, for all neighbourhoods $V$ with $E \subseteq V$, there exists $U$ such that $E \subseteq U \subseteq V$ and for all $x \in U$, and for some solution $\xi_{t}$ of $(10)$ with $\xi_{0}(x)=x$,

1. $\xi_{t}(x) \in V, \forall t \geq 0$ and

2. $\lim _{t \rightarrow \infty} \xi_{t}(x) \in E$

\section{Remarks}

- Hence for an asymptotically stable equilibrium set $E$, any equilibrium point $y \in E$ is weakly asymptotically stable in the above sense.

- The above definitions are also valid for invariant sets. In this way, we can define the stability of $\Pi(D)$.

The non-uniqueness of solutions to the differential inclusion also gives rise to two different notions of instability.

Definition 10. An equilibrium set $E$ is weakly unstable if there exists a neighbourhood $V$ with $E \subseteq V$, such that for all neighbourhoods $U$ with $E \subseteq U \subseteq V$, there exist some $x \in U$, some $\tau>0$, such that for some solution $\xi_{t}$ of $(10)$, with $\xi_{0}(x)=x$

$$
\xi_{\tau}(x) \notin V .
$$

Definition 11. An equilibrium set $E$ is unstable if there exists a neighbourhood $V$ with $E \subseteq V$, such that for all neighbourhoods $U$ with $E \subseteq U \subseteq V$, there exist some $x \in U$, some $\tau>0$, such that for all solutions $\xi_{t}$ of $(10)$, with $\xi_{0}(x)=x$

$$
\xi_{\tau}(x) \notin V .
$$


Remark. Definitions 10 and 11 have been formulated so that weakly unstable is equivalent to not stable, and unstable is equivalent to not weakly stable. Hence if an equilibrium set $E$ is weakly unstable, it follows that $E$ is not stable, but it may be weakly stable. If an equilibrium set $E$ is unstable, it follows $E$ is also weakly unstable.

\section{Graphical Representation of the Dynamics}

De Jong et al have developed a discrete, qualitative description of the dynamics of the PL system that underlies the qualitative simulation of genetic regulatory networks [dJGH $\left.{ }^{+} 04\right]$. This formulation involves an abstraction of the dynamics of the system by means of a state transition graph, a concept originally due to Glass [Gla75]. The state transition graph is a discrete representation consisting of the qualitative states of the system (the domains) and all possible transitions between them. This description is key to formulating and proving the results on stability of singular equilibrium points in Section 5, so here we review some of the concepts and prove a proposition on transitions that will be used in the proofs.

\subsection{States and Transitions}

The set of domains $\mathcal{D}$ can be thought of as qualitative states, since the PL system (2) behaves in a qualitatively homogeneous manner in each domain $D \in \mathcal{D}$. The qualitative state associated with a domain can be either persistent (some solutions remain in the domain for more than a single instant of time) or instantaneous (all solutions pass through the domain instantaneously), the latter being of limited biological significance. Given two contiguous domains $D, D^{\prime} \in \mathcal{D}$, we say that there is transition from $D$ to $D^{\prime}$ if there is a solution in the sense of Filippov lying in $D$ and terminating in $D^{\prime}$. We define a transition from $D$ to $D^{\prime}$ more precisely below. Recall that $A(D) \subset \mathcal{D}_{s}$ is the set of all domains in the boundary of $D$.

Definition 12. Let $D, D^{\prime} \in \mathcal{D}$ be two contiguous domains. We say that there exists a transition from $D$ to $D^{\prime}$ if one of the following two properties holds:

1. If $D^{\prime} \in A(D)$, then there exists $x_{0} \in D$ and a Filippov solution $\xi_{t}\left(x_{0}\right)$ defined on a finite time interval $[0, \tau]$ such that

(a) $\xi_{t}\left(x_{0}\right) \in D$ for all $t \in[0, \tau)$, and

(b) $\xi_{\tau}\left(x_{0}\right) \in D^{\prime}$.

2. If $D \in A\left(D^{\prime}\right)$, then there exists $x_{0} \in D$ and a Filippov solution $\xi_{t}\left(x_{0}\right)$ defined on a finite time interval $[0, \tau]$ such that

(a) $\xi_{0}\left(x_{0}\right)=x_{0} \in D$, and

(b) $\xi_{t}\left(x_{0}\right) \in D^{\prime}$ for all $t \in(0, \tau]$. 
The definition says that there exists a Filippov solution reaching $D^{\prime}$ from $D$ in finite time without passing through an intermediate domain. Given contiguous $D$ and $D^{\prime}$, then either $D^{\prime} \subseteq \partial D$ (that is, $D^{\prime} \in A(D)$ ), or $D \subseteq \partial D^{\prime}$ (that is, $D \in A\left(D^{\prime}\right)$ ). If $D^{\prime} \subseteq \partial D$ then $\operatorname{order}\left(D^{\prime}\right)>\operatorname{order}(D)$, meaning $D^{\prime}$ has more switching variables then $D$. Alternatively, if $D \subseteq \partial D^{\prime}$ then $\operatorname{order}\left(D^{\prime}\right)<\operatorname{order}(D)$, i.e. $D^{\prime}$ has less switching variables than $D$. Based on the order of the two domains, we can prove the following two properties of transitions.

Proposition 4.1. Let $D, D^{\prime} \in \mathcal{D}$ be two contiguous domains such that $D^{\prime} \in A(D)$. Under Assumption 1, there exists a transition from $D$ to $D^{\prime}$ iff

1. $\Phi(D) \neq\{\}$

2. For all $i \in\{1, \ldots, n\}$ such that $x_{i}$ is switching in $D^{\prime}$ but not in $D$,

$$
\left(d^{\prime}{ }_{i}-d_{i}\right)\left(\phi_{i}-d^{\prime}{ }_{i}\right)>0, \forall d \in D, \forall d^{\prime} \in D^{\prime}, \forall \phi \in \Phi(D) .
$$

Proof: We first prove sufficiency. Suppose $\Phi(D) \neq\{\}$. Let $x_{i}$ be switching in $D^{\prime}$ but not in $D$. Then we can write $D^{\prime}=D^{\prime}{ }_{1} \times \cdots \times D^{\prime}{ }_{i} \times \cdots \times D^{\prime}{ }_{n}$, with $D^{\prime}{ }_{i}=\left\{x_{i} \in \Omega_{i} \mid x_{i}=\theta_{i}^{q_{i}}\right\}$. Assume initially that $d_{i}<\theta_{i}^{q_{i}}, \forall d \in D$. Then $d^{\prime}{ }_{i}-d_{i}>0, \forall d^{\prime} \in D^{\prime}, \forall d \in D$. Thus condition 2 above yields $\phi_{i}-d^{\prime}{ }_{i}>0$ for all $\phi \in \Phi(D)$, so $d^{\prime}{ }_{i}<\phi_{i}$. Hence $d_{i}<\theta_{i}^{q_{i}}<\phi_{i}, \forall d \in D$. Similarly, if $d_{i}>\theta_{i}^{q_{i}}, \forall d \in D$, it follows that $d_{i}>\theta_{i}^{q_{i}}>\phi_{i}, \forall d \in D$. See Figure 5(a) for an illustration. For every $x_{0} \in D$, there exists a solution $\xi_{t}$ such that $\left(\xi_{t}\left(x_{0}\right)\right)_{i}$ converges monotonically towards the projection of $\Phi(D)$ onto $\Omega_{i}$, by Lemma 2.1. Hence all solutions starting in $D$ will reach the hyperplane $\left\{x \in \Omega \mid x_{i}=\theta_{i}^{q_{i}}\right\}$, and do so in finite time by Assumption 1. Let $I$ be the set of indices such that $x_{i}$ is switching in $D^{\prime}$ but not in $D$. Then choose $x_{0}$ so that the solution $\xi_{t}\left(x_{0}\right)$ passes through

$$
\bigcap_{i \in I}\left\{x \in \Omega \mid x_{i}=\theta_{i}^{q_{i}}\right\} \cap \partial D .
$$

According to Definition 12, this gives a transition from $D$ to $D^{\prime}$.

To prove necessity, we use a proof by contradiction. Assume conditions 1 or 2 are false and suppose there exists a transition from $D$ to $D^{\prime} \in A(D)$. Firstly, if condition 1 is false then $\Phi(D)$ is empty, no solutions stay in $D$, so no transition from $D$ to $D^{\prime} \in A(D)$ is possible according to Definition 12. Secondly, if condition 2 is false then under Assumption 1, there exists $i$ with $x_{i}$ switching in $D^{\prime}$ but not in $D$ such that

$$
\left(d^{\prime}{ }_{i}-d_{i}\right)\left(\phi_{i}-d^{\prime}{ }_{i}\right)<0 \forall \phi \in \Phi(D), \forall d \in D, \forall d^{\prime} \in D^{\prime} .
$$

Note that we can write (20) with a strict inequality since under Assumption $1, \Phi(D) \cap$ $D^{\prime}=\{\}$, so $\phi_{i} \neq d_{i}^{\prime}$. In addition, $d^{\prime}{ }_{i} \neq d_{i}$ by the definition of domains. Assume that $D^{\prime}{ }_{i}=\left\{x_{i} \in \Omega_{i} \mid x_{i}=\theta_{i}^{q_{i}}\right\}$. Thus $d^{\prime}{ }_{i}=\theta_{i}^{q_{i}}$. If $d^{\prime}{ }_{i}<d_{i}$, then this implies $\phi_{i}>d^{\prime}{ }_{i}$. Similarly, if $d^{\prime}{ }_{i}>d_{i}$ then $\phi_{i}<d^{\prime}{ }_{i}$. From Lemma 2.1 we know that for all solutions $\xi_{t}$ in $D$, the $i$ component $\left(\xi_{t}\right)_{i}$ converges monotonically to the projection of $\Phi(D)$ onto $\Omega_{i}$. For both cases, it follows that no solution starting in $D$ can reach the threshold hyperplane 


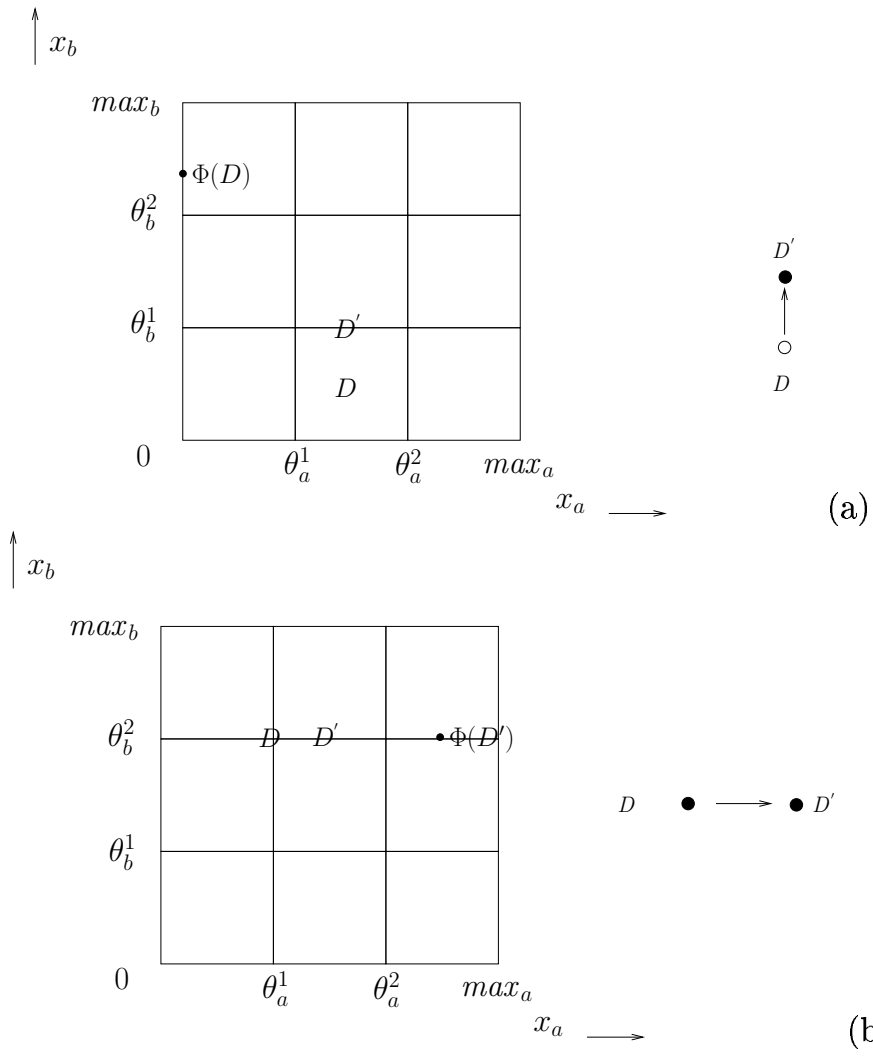

Figure 5: Typical phase portrait and subgraph of the state transition graph for Propositions 4.1 and 4.2. (a) For Proposition 4.1, $\operatorname{order}\left(D^{\prime}\right)>\operatorname{order}(D), x_{b}$ is switching on $D^{\prime}$ and the location of $\Phi(D)$ results in a transition from $D$ to $D^{\prime}$. (b) For Proposition 4.2, $\operatorname{order}\left(D^{\prime}\right)<\operatorname{order}(D), x_{a}$ is switching in $D^{\prime}$, but both $x_{a}$ and $x_{b}$ are switching on $D$. The location of $\Phi\left(D^{\prime}\right)$ guarantees a transition from $D$ to $D^{\prime}$. Regulatory and switching domains are indicated by unfilled and filled dots, respectively.

$\left\{x \in \Omega \mid x_{i}=d^{\prime}{ }_{i}\right\}$. Hence there cannot be a transition from $D$ to $D^{\prime}$, which contradicts our assumption.

Proposition 4.2. Let $D, D^{\prime} \in \mathcal{D}$ be two contiguous domains such that $D \in A\left(D^{\prime}\right)$. Under Assumption 1, there exists a transition from $D$ to $D^{\prime}$ iff

1. $\Phi\left(D^{\prime}\right) \neq\{\}$

2. For all $i \in\{1, \ldots, n\}$ such that $x_{i}$ is switching in $D$ but not in $D^{\prime}$,

$$
\left(d^{\prime}{ }_{i}-d_{i}\right)\left(\phi_{i}^{\prime}-d_{i}\right)>0, \forall d \in D, \forall d^{\prime} \in D^{\prime}, \forall \phi^{\prime} \in \Phi\left(D^{\prime}\right) .
$$

$\mathrm{RR} \mathrm{n}^{\circ} 5353$ 
Proof: Similar to the proof of Proposition 4.1.

Typical situations for Propositions 4.1 and 4.2 are illustrated in Figure 5. In Figure 5(a), we have $D^{\prime} \in A(D), \operatorname{order}\left(D^{\prime}\right)>\operatorname{order}(D), x_{b}$ is switching on $D^{\prime}$ and the relative location of $\Phi(D)$ leads to a transition from $D$ to $D^{\prime}$, as covered by Proposition 4.1. In Figure 5(b), $D \in A\left(D^{\prime}\right)$, order $\left(D^{\prime}\right)<\operatorname{order}(D), x_{a}$ is switching in $D^{\prime}$, and both $x_{a}$ and $x_{b}$ are switching on $D$. The relative location of $\Phi\left(D^{\prime}\right)$ leads to a transition from $D$ to $D^{\prime}$, as covered by Proposition 4.2.

\subsection{State Transition Graph}

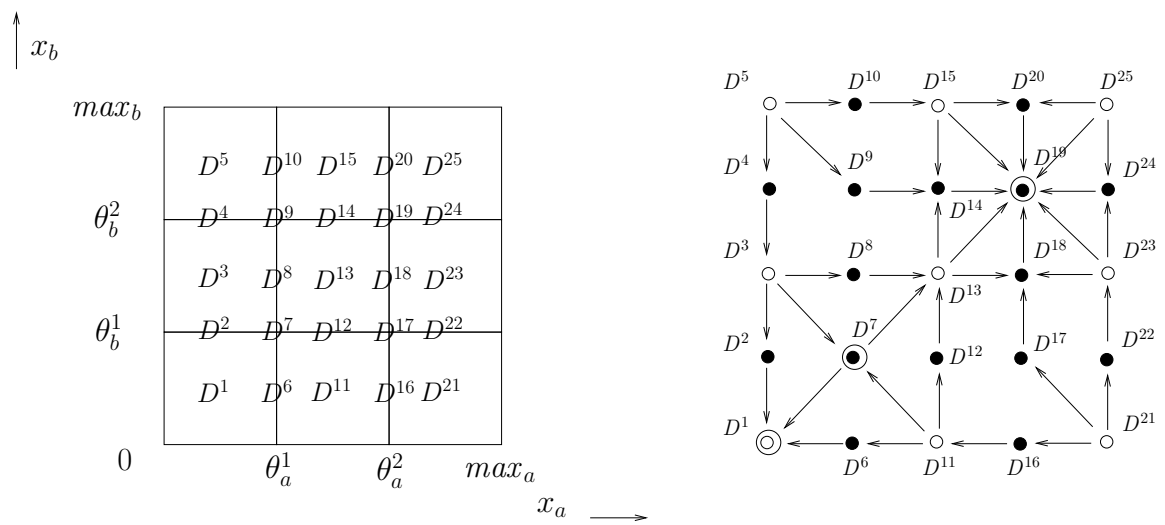

Figure 6: Phase space and state transition graph for the PL model in Figure 1. Nodes associated with regulatory and switching domains are indicated by unfilled and filled dots, respectively. Domains containing equilibria are additionally circled.

A state transition graph (STG) is a directed graph whose vertices are the domains of the system and whose edges are the transitions between these domains. The state transition graph is a discrete representation of the qualitative dynamics of the piecewise-linear system. Figure 6 shows the state transition graph for the example two-gene network from Figure 1. The domains represented by the nodes in the state transition graph can be thought of as qualitative states of the PL model. We have the following definitions for paths in a state transition graph $G$.

Definition 13. A path of length $(k-1)$ in $G$ is a non-empty sequence of domains $\left\langle D_{1}, \ldots, D_{k}\right\rangle$ such that, for every $i, 1 \leq i<k$, there exists a transition from $D_{i}$ to $D_{i+1}$.

Definition 14. A cycle in $G$ is a path that begins and ends with the same domain (i.e. the same vertex), but otherwise all the domains are different.

Recall that within each domain, i.e. for each vertex of the STG, the PL system (2) behaves in a qualitatively homogeneous way. Hence many dynamical properties of the full PL

INRIA 
system can be analyzed simply by studying the STG. In the terminology of Definitions 13 and 14, a solution to the PL system (2) corresponds to a path in the STG and a cycle in the PL system will show up as a cycle in the STG. Since the STG captures the essential qualitative dynamics of the PL system, it is useful to prove results on stability of equilibria that can be inferred directly from the STG. For example, for the two-gene network, the sequence $\left\langle D^{21}, D^{17}, D^{18}, D^{19}\right\rangle$ is path in the STG shown in Figure 6. This path corresponds to solutions with initial conditions in domain $D^{21}$ that reach a qualitative equilibrium state in $D^{19}$, corresponding to a domain containing an equilibrium point. Whether such qualitative equilibrium states represent equilibria that are stable, according to Definitions $6-9$, is investigated in the next section.

\section{Criteria for Stability of Singular Equilibria}

The purpose of this section is to set out and prove criteria for the stability of singular equilibria of the differential inclusion (10), based on properties of the state transition graph. As discussed in Section 3, regular equilibrium points are known to be asymptotically stable so we focus instead on the stability of equilibria located in switching domains: singular equilibrium points and sets. We work under the caveat that Assumption 1 from Section 2.3 holds for every domain $D$ in our system. In particular this excludes the situation when the focal set $\Phi(D)$ is only partially contained in $D \in \mathcal{D}_{s}$ and spills over into contiguous switching domains, or the situation when $\Phi(D)$ is contained in the supporting hyperplanes of the boundary of $D$. The proof in $\mathbb{R}^{2}$ is included since it gives a geometrical intuition for the general proof in $\mathbb{R}^{n}$.

\subsection{Theorem on Stability}

The following theorems allow us to link the rigorous concept of stability seen in Definitions 69 with the qualitative dynamics of the system represented by the state transition graph. Theorems 5.1 and 5.2 state that a switching domain $D$ contains an asymptotically stable equilibrium set if every contiguous regulatory domain has a transition entering the domain $D$. The first theorem is stated and proved for $\Omega \subset \mathbb{R}^{2}$, and the second is the general case for $p$ switching variables in $\Omega \subset \mathbb{R}^{n}$.

Theorem 5.1. Assume $\Omega \subset \mathbb{R}^{2}$. Let $D \in \mathcal{D}_{\text {s }}$ be a switching domain containing a singular equilibrium point $\Phi(D)$. If for all $D^{\prime} \in R(D)$ there exists a transition from $D^{\prime}$ to $D$ in the state transition graph $G$, then $\Phi(D)$ is asymptotically stable.

Proof: Let $x=\left(x_{1}, x_{2}\right) \in D$. In $\mathbb{R}^{2}$ there are only two cases: one of the variables is switching, or both are switching.

1. Assume $x_{2}$ is a switching variable on $D$ and $x_{1}$ is not. The subgraph of $G$ showing $D$ and $R(D)$ can be seen in Figure 7. Assume without loss of generality that $D$ is defined by $D=\left\{x \in \Omega \mid \theta_{1}^{q_{1}}<x_{1}<\theta_{1}^{q_{1}+1}, x_{2}=\theta_{2}^{q_{2}}\right\}$. Let $D^{1}=\left\{x \in \Omega \mid \theta_{1}^{q_{1}}<x_{1}<\right.$ 


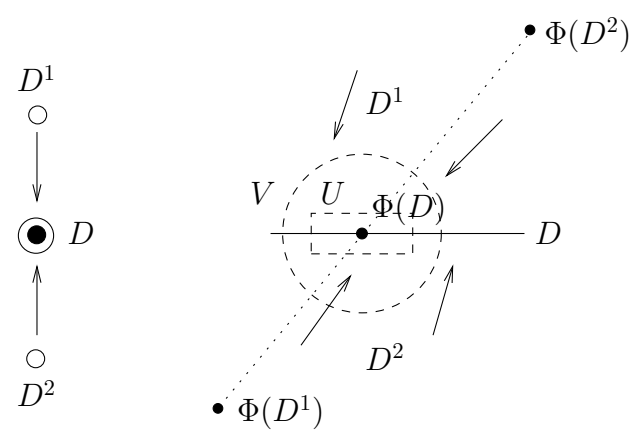

Figure 7: State transition graph and sketch of phase portrait for case 1 in the proof of Theorem 5.1.

$\left.\theta_{1}^{q_{1}+1}, \theta_{2}^{q_{2}}<x_{2}<\theta_{2}^{q_{2}+1}\right\}$ and $D^{2}=\left\{x \in \Omega \mid \theta_{1}^{q_{1}}<x_{1}<\theta_{1}^{q_{1}+1}, \theta_{2}^{q_{2}-1}<x_{2}<\theta_{2}^{q_{2}}\right\}$ be the regulatory domains in $R(D)$ i.e. $D^{1}$ and $D^{2}$ are the domains "above" and "below" $D$ in phase space. Since there is a transition from $D^{1}$ to $D$ in the STG, it follows from Proposition 4.1 that

$$
\left(d_{2}-d_{2}^{1}\right)\left(\phi_{2}^{1}-d_{2}\right)>0, \forall d \in D, \forall d^{1} \in D^{1},
$$

where $\phi^{1}=\phi\left(D^{1}\right)$. Since $d_{2}<d_{2}^{1}, \forall d \in D, \forall d^{1} \in D^{1}$, we know that the focal point $\phi\left(D^{1}\right)$ lies somewhere in the half-space defined by $\left\{x \in \Omega \mid x_{2}<\theta_{2}^{q_{2}}\right\}$. That is, $\phi\left(D^{1}\right)$ lies "below" the threshold defined by $x_{2}=\theta_{2}^{q_{2}}$. This means that every solution $\xi_{t}=\left(x_{1}(t), x_{2}(t)\right)$ starting in $D^{1}$, has the property $\dot{x}_{2}<0$. Similarly, the transition from $D^{2}$ to $D$ in the STG and Proposition 4.1 imply $\phi\left(D^{2}\right)$ lies somewhere in the half-space defined by $\left\{x \in \mathbb{R}^{2} \mid x_{2}>\theta_{2}^{q_{2}}\right\}$, so every solution $\xi_{t}=\left(x_{1}(t), x_{2}(t)\right)$ starting in $D^{2}$ has $\dot{x}_{2}>0$. Moreover, we can always bound the non-switching component of the solutions, $x_{1}(t)$, using bounds of order $e^{-\gamma_{1} t}$ w.r.t. time. This means that given any neighbourhood $V$ of $\Phi(D) \in D$, we can choose another neighbourhood $U$ of $\Phi(D)$ with $U$ small enough such that $\xi_{t}(x) \in V, \forall t>0, \forall x \in U$, and that all solutions $\xi_{t}$ starting in $U$ reach $D$ in finite time. More exactly, let $\Phi(D)=\left\{\left(c, \theta_{2}^{q_{2}}\right)\right\}$, and choose $U$ to be the neighbourhood

$$
U=\left\{x \in \Omega|| x_{1}-c\left|<\varepsilon_{1},\right| x_{2}-\theta_{2}^{q_{2}} \mid<\varepsilon_{2}\right\},
$$

for some $\varepsilon_{1}, \varepsilon_{2}>0$. From Lemma 2.1, the monotonicity properties of the solutions to (2) mean that given any neighbourhood $V$, we can always choose $\varepsilon_{1}, \varepsilon_{2}$ small enough so that $U \subset V$ and $\xi_{t}(x) \in V, \forall t>0, \forall x \in U$. (Briefly, the appropriate values of $\varepsilon_{1}, \varepsilon_{2}$ can be chosen by computing the times $t^{ \pm}$to reach $x_{2}=\theta_{2}^{q_{2}}$ from $x_{2}=\theta_{2}^{q_{2}} \pm \varepsilon_{2}$ using the $\dot{x}_{2}$ equation, calculating $x_{1}\left(t^{ \pm}\right)$, and choosing $\varepsilon_{1}, \varepsilon_{2}$ so that $\left(x_{1}\left(t^{ \pm}\right), x_{2}\left(t^{ \pm}\right)\right) \in V$ for all initial conditions in $U$.) Thus for small enough $U$, all solutions starting in $U$ are guaranteed to reach $D$ (in finite time because of Assumption 1) and when in $D$, they are known to converge asymptotically to $\Phi(D)$ [GS02]. 

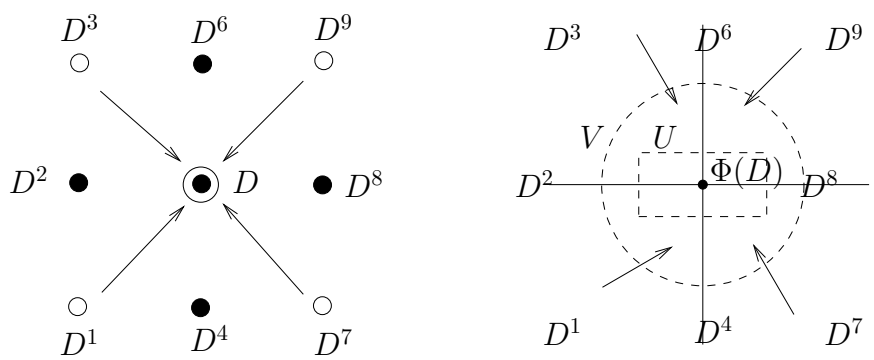

Figure 8: State transition graph and sketch of phase portrait for case 2 in the proof of Theorem 5.1. Only transitions from $D^{\prime} \in R(D)$ are shown.

2. Assume both $x_{1}$ and $x_{2}$ are switching variables on $D$. Then there are four regulatory domains in $R(D)$ and we have the situation shown in Figure 8. For each regulatory domain $D^{\prime} \in R(D)=\left\{D^{1}, D^{3}, D^{7}, D^{9}\right\}$, we can extend the arguments used in case 1 above to deduce that $\phi\left(D^{i}\right)(i=1,3,7,9)$ lie in a specific quadrant. For example, the transition from $D^{3}$ to $D$ and Proposition 4.1 implies that $\phi\left(D^{3}\right)$ lies in the quarterspace defined by $\left\{x \in \Omega \mid x_{1}>\theta_{1}^{q_{1}}, x_{2}<\theta_{2}^{q_{2}}\right\}$. This can be seen as follows. Let $D=$ $\left\{x \in \Omega \mid x_{1}=\theta_{1}^{q_{1}}, x_{2}=\theta_{2}^{q_{2}}\right\}, D^{3}=\left\{x \in \Omega \mid \theta_{1}^{q_{1}-1}<x_{1}<\theta_{1}^{q_{1}}, \theta_{2}^{q_{2}}<x_{2}<\theta_{2}^{q_{2}+1}\right\}$, and $D^{7}=\left\{x \in \Omega \mid \theta_{1}^{q_{1}}<x_{1}<\theta_{1}^{q_{1}+1}, \theta_{2}^{q_{2}-1}<x_{2}<\theta_{2}^{q_{2}}\right\}$. Then $D^{3}$ and $D^{7}$ are regulatory domains as illustrated in Figure 8. By hypothesis, there is a transition from $D^{3}$ to $D$ in the STG, so it follows from Proposition 4.1 that

$$
\begin{aligned}
& \left(d_{1}-d_{1}^{3}\right)\left(\phi_{1}^{3}-d_{1}\right)>0, \forall d \in D, \forall d^{3} \in D^{3}, \\
& \left(d_{2}-d_{2}^{3}\right)\left(\phi_{2}^{3}-d_{2}\right)>0, \forall d \in D, \forall d^{3} \in D^{3},
\end{aligned}
$$

where $\phi^{3}=\phi\left(D^{3}\right)$. Since $d_{2}<d_{2}^{3}, \forall d \in D, \forall d^{3} \in D^{3},(24)$ implies that the focal point $\phi\left(D^{3}\right)$ lies somewhere in the half-space defined by $\left\{x \in \Omega \mid x_{2}<\theta_{2}^{q_{2}}\right\}$. Similarly, since $d_{1}^{3}<d_{1}, \forall d \in D, \forall d^{3} \in D^{3}$, (23) implies that the focal point $\phi\left(D^{3}\right)$ lies somewhere in the half-space defined by $\left\{x \in \Omega \mid x_{1}>\theta_{1}^{q_{1}}\right\}$. Thus, (23) and (24) together imply that

$$
\phi\left(D^{3}\right) \in\left\{x \in \Omega \mid x_{1}>\theta_{1}^{q_{1}}\right\} \cap\left\{x \in \Omega \mid x_{2}<\theta_{2}^{q_{2}}\right\},
$$

and every solution, $\xi_{t}=\left(x_{1}(t), x_{2}(t)\right)$, starting in $D^{3}$ has the property $\dot{x}_{1}>0$ and $\dot{x}_{2}<0$. Taking a sector $U^{3}$ in $D^{3}$ that will form part of a neighbourhood of $\Phi(D)$, all solutions starting in $U^{3}$ will either reach one of the switching domains $D^{2}$ or $D^{6}$ or go directly to $D$. The transition from $D^{3}$ and $D^{1}$ to $D$ in the STG result in sliding modes along $D^{2}$, with focal set $\Phi\left(D^{2}\right)$ such that $\Phi\left(D^{2}\right) \subseteq\left\{x \in \Omega \mid x_{1}>\theta_{1}^{q_{1}}\right\}$. This is because $\Phi\left(D^{2}\right)$ is defined using a convex combination of $\phi\left(D^{1}\right)$ and $\phi\left(D^{3}\right)$, both of which lie in the region $\Phi\left(D^{2}\right) \subseteq\left\{x \in \Omega \mid x_{1}>\theta_{1}^{q_{1}}\right\}$, hence $\Phi\left(D^{2}\right)$ will also lie in this region. It follows that solutions starting in $U^{3}$ that reach $D^{2}$ will slide along $D^{2}$ until they reach $D$. Similarly, solutions starting in $U^{3}$ that reach $D^{6}$ will slide along $D^{6}$ until they reach $D$. Analogous regions can be constructed in the other regulatory domains, 
$\left\{D^{1}, D^{7}, D^{9}\right\}$. From these sectors, $\left\{U^{1}, U^{3}, U^{7}, U^{9}\right\}$, in the domains $\left\{D^{1}, D^{3}, D^{7}, D^{9}\right\}$, we can construct a neighbourhood $U$ of $\Phi(D)$. Given any neighbourhood $V$ of $\Phi(D)$, we can construct such a neighbourhood $U \subset V$. From Lemma 2.1, the monotonic convergence of solutions means that all solutions starting in a small enough $U$ will remain in $V$. The above reasoning shows that all these solutions eventually converge to $D$ and hence to $\Phi(D)$.

This completes the proof of Theorem 5.1.

Remark. The proof of Theorem 5.1 is essentially an application of Proposition 4.1 (under Assumption 1) and Lemma 2.1. We use Proposition 4.1 to deduce, from the existence of a transition, the relative position of the focal points for each $D^{\prime} \in R(D)$, construct a suitable neighbourhood around $\Phi(D)$ and then apply Lemma 2.1, on the monotone convergence of solutions in $D^{\prime}$ towards $\Phi\left(D^{\prime}\right)$, to prove that $\Phi(D)$ is asymptotically stable according to Definition 8. Assumption 1 ensures that all solutions starting in the neighbourhood will reach $D$ in finite time, and is used implicitly when Proposition 4.1 is applied.

Below we state and prove the general result in $\mathbb{R}^{n}$ for $p$ switching variables. We give the main lines, the details being similar to the two dimensional case.

Theorem 5.2. Assume $\Omega \subset \mathbb{R}^{n}$. Let $D \in \mathcal{D}_{s}$ be a switching domain of order $p>1$ containing a singular equilibrium set $\Phi(D)$ that satisfies Assumption 1. If for all $D^{\prime} \in$ $R(D)$, there is a transition from $D^{\prime}$ to $D$ in the state transition graph, then $\Phi(D)$ is weakly asymptotically stable and $\Pi(D)$ is asymptotically stable.

\section{Proof:}

Assume without loss of generality that $x_{1}, \ldots, x_{p}$ are the switching variables on $D$ and $x_{p+1}, \ldots, x_{n}$ are non-switching. Assume then that $D$ is defined by

$$
D=\left\{x \in \Omega \mid x_{1}=\theta_{1}^{q_{1}}, \ldots, x_{p}=\theta_{p}^{q_{p}}, \theta_{p+1}^{q_{p+1}}<x_{p+1}<\theta_{p+1}^{q_{p+1}+1}, \ldots, \theta_{n}^{q_{n}}<x_{n}<\theta_{n}^{q_{n}+1}\right\} .
$$

Let $D^{\prime} \in R(D)$ be a contiguous regulatory domain. Let $i \in\{1, \ldots, p\}$ index an arbitrary variable that is switching in $D$ but not in $D^{\prime}$ (since $D^{\prime}$ is a regulatory domain). From the hypotheses of the theorem, there exists a transition from $D^{\prime}$ to $D$. Since $D^{\prime}$ is a regulatory domain, we know

$$
p=\operatorname{order}(D)>\operatorname{order}\left(D^{\prime}\right)=0
$$

and that $\Phi\left(D^{\prime}\right) \neq\{\}$. In fact, since $D^{\prime} \in \mathcal{D}_{r}$, we can write $\Phi\left(D^{\prime}\right)=\left\{\phi\left(D^{\prime}\right)\right\}$. Hence from Proposition 4.1 on transitions, and under Assumption 1, for each $i$ with $x_{i}$ switching in $D$, we have

$$
\left(d_{i}-d^{\prime}{ }_{i}\right)\left(\phi_{i}^{\prime}-d_{i}\right)>0, \forall d^{\prime} \in D^{\prime}, \forall d \in D
$$

where $\phi^{\prime}=\phi\left(D^{\prime}\right)$. Hence either we have

$$
0 \leq d^{\prime}{ }_{i}<d_{i}<\phi_{i}^{\prime}, \forall d^{\prime} \in D^{\prime}, \forall d \in D,
$$


which means $\phi\left(D^{\prime}\right)$ lies somewhere in the region $\left\{x \in \Omega \mid x_{i}>\theta_{i}^{q_{i}}\right\}$, or we have

$$
0 \leq \phi_{i}^{\prime}<d_{i}<d^{\prime}{ }_{i}, \forall d^{\prime} \in D^{\prime}, \forall d \in D,
$$

which means $\Phi\left(D^{\prime}\right)$ lies somewhere in the region $\left\{x \in \Omega \mid x_{i}<\theta_{i}^{q_{i}}\right\}$. If equation (28) is true for some $D^{\prime} \in R(D)$, then every solution $\xi_{t}=\left(x_{1}(t), x_{2}(t), \ldots, x_{n}(t)\right)$ starting in $D^{\prime}$ has the property $\dot{x}_{i}>0$ in $D^{\prime}$. Similarly, equation (29) means that for every solution $\xi_{t}=\left(x_{1}(t), x_{2}(t), \ldots, x_{n}(t)\right)$ starting in $D^{\prime}$, we have the property $\dot{x}_{i}<0$ in $D^{\prime}$. This is true for any $i$ with $x_{i}$ switching in $D^{\prime}$, thus for all switching variables $x_{1}, \ldots, x_{p}$. In addition, as seen in Theorem 5.1, case 1, we can always bound the non-switching components of the solutions by bounds of order $e^{-\gamma_{j} t}$ w.r.t. time, for each of the non-switching variables $x_{p+1}, \ldots, x_{n}$. From Lemma 2.1, the solutions $\xi_{i}$ in $D^{\prime} \in D_{r}$ converge monotonically towards $\phi\left(D^{\prime}\right)$. Hence, given any neighbourhood $V$ of $\Phi(D)$, we can bound the solutions by choosing another neighbourhood $U$ containing $\Phi(D)$, with $U$ small enough such that $\xi_{t}(x) \in V$, $\forall t>0, \forall x \in U$, and such that all solutions starting in $U$ reach $D$ in finite time. This neighbourhood $U$ can be constructed analogously to the neighbourhoods in the proof of Theorem 5.1, being careful to include sectors on the contiguous switching domains. The focal sets of these contiguous switching domains are derived from convex combinations of the focal points in the neighbouring regulatory domains, so the solutions in the switching domains have the same properties of monotonicity. Once the solutions reach $D$ they converge asymptotically to $\Pi(D)$ (Corollary 1 ), and so $\Pi(D)$ is an asymptotically stable equilibrium set.

Let us now consider the equilibrium set $\Phi(D)$; every $\phi \in \Phi(D)$ is a singular equilibrium for the system, so there is, for an initial condition $x\left(t_{0}\right)$, a solution (among all the solutions starting in $\left.x\left(t_{0}\right)\right)$ in $D$ verifying $\dot{x}=\gamma(\phi-x)$. This solution converges towards $\phi$. Now it is easy to construct a neighborhood of $\Phi(D)$ having the required properties for weak asymptotic stability: choosing a neighborhood $V$ of $\Phi(D)$, we wish to construct a neighborhood $U \subseteq V$ verifying Definition 9. For every $\phi \in \Phi(D)$, choose a hyperrectangular neighborhood of $\phi$ contained in $V$. Clearly this neighborhood is invariant for the solution converging towards $\phi$. Let us take the union of these neighborhoods for all $\phi \in \Phi(D)$, we obtain a neighborhood $U$ of $\Phi(D)$ proving the weak asymptotic stability.

Corollary 4. Under the conditions of 5.2, if, moreover, $\Phi(D)$ is a point, it is asymptotically stable.

Remark. Because of the linearity of the differential system for a given $\phi \in \Phi(D)$, it is also clear that the basins of attraction for $\Phi(D)$ and $\Pi(D)$ include the whole domain $D$.

There is no hope for proving more in the case when $\Phi(D)$ is a set. To see that, let us suppose that $n=4$, underwhich condition $\Phi(D)$ can be a segment when $D$ is of dimension 2. If this segment is not parallel to the axes, and if the $\gamma_{i}$ are not all equal, then there exist solutions starting from one point $\phi_{1}$ of the segment and leaving the segment (but staying in the rectangle $\Pi(D))$ to converge towards another point $\phi_{2}$ of the segment (Fig. 9).

In the two-gene network of Figure 1, the switching domain $D^{19}$ satisfies the criteria of Theorems 5.1 or 5.2 , since each domain $D^{\prime} \in R(D)=\left\{D^{13}, D^{15}, D^{23}, D^{25}\right\}$ has a transition 


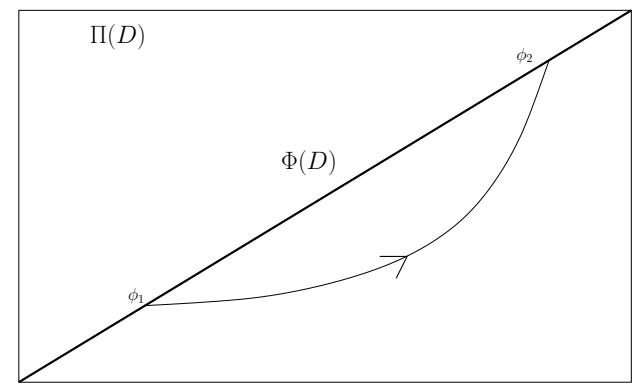

Figure 9: Illustration of the fact that $\Phi(D)$ is weakly asymptotically stable but not asymptotically stable in the sense of definition 6 .

from $D^{\prime}$ to $D^{19}$. Hence Theorems 5.1 or 5.2 show that $D^{19}$ contains an asymptotically stable singular equilibrium point.

\subsection{Theorem on Instability}

The final theorem, Theorem 5.3, is a result for unstable singular equilibrium sets, like the saddle-type behaviour of $D^{7}$ in the example network, Figure 6 . It states that a domain $D$ contains an unstable equilibrium set if there is at least one transition in the state transition graph leaving $D$ and entering a contiguous regulatory domain.

Theorem 5.3. Let $\Omega \subset \mathbb{R}^{n}$ and $D \in \mathcal{D}_{s}$ be a switching domain containing a singular equilibrium set $\Phi(D) \subseteq D$ that satisfies Assumption 1. If there exists $D^{\prime} \in R(D)$ with a transition from $D$ to $D^{\prime}$, then $\Phi(D)$ is unstable.

Proof: First consider the case $n=2$. Since $\Omega \subset \mathbb{R}^{2}$, it follows that $\Phi(D)$ is a singleton, $\Phi(D)=\{\phi\}$ with $\phi \in D$. First suppose $x_{1}$ is switching on $D$, that the regulatory domain $D^{\prime} \in R(D)$ is given by $D^{\prime}=\left\{x \in \Omega \mid \theta_{1}^{q_{1}}<x_{1}<\theta_{1}^{q_{1}+1}, \theta_{2}^{q_{2}}<x_{2}<\theta_{2}^{q_{2}+1}\right\}$, and that $D$ is given by $D=\left\{x \in \Omega \mid x_{1}=\theta_{1}^{q_{1}}, \theta_{2}^{q_{2}}<x_{2}<\theta_{2}^{q_{2}+1}\right\}$. Since there is a transition from $D$ to $D^{\prime}$, we can deduce from Proposition 4.2 that the focal point $\Phi\left(D^{\prime}\right)$ lies in the region $\left\{x \in \Omega \mid x_{1}>\theta_{1}^{q_{1}}\right\}$. Choose a neighbourhood $V$ of $\phi$ given by $B(\phi, r)$, where $B(y, r)$ is the 2-ball of radius $r>0$ and centre $y$. Then for any neighbourhood $U$ of $\phi$, with $U \subset V$, by the monotonic convergence of the solutions in $D^{\prime}$ towards $\Phi\left(D^{\prime}\right)$ (from Lemma 2.1), we can always find some $x_{0} \in U$ and a solution $\xi_{t}$ such that $\xi_{\tau}\left(x_{0}\right) \notin V$ for some $\tau>0$. Indeed if $V=B(\phi, r)$ is chosen with $r$ small enough then any $x_{0}$ in $\left(U \cap D^{\prime}\right)$ will work since $\xi_{t}\left(x_{0}\right)$ will converge towards $\Phi\left(D^{\prime}\right)$, away from $D$, and leave $V$. The proof when both $x_{1}$ and $x_{2}$ are switching on $D$ is similar, and this proof can be easily generalized to $\mathbb{R}^{n}$.

The above proof works by taking a neighbourhood $V$ around the equilibrium set, and showing that for all other neighbourhoods contained in $V$, we have solutions that leave $V$ in finite time (cf. Figure(10)). In fact, the transition from $D$ to $D^{\prime}$ and Proposition 4.2 mean that we can always take a solution starting in $D^{\prime}$, which will converge monotonically 
towards $\Phi\left(D^{\prime}\right)$ (Lemma 2.1), thus leaving $V$ in finite time.

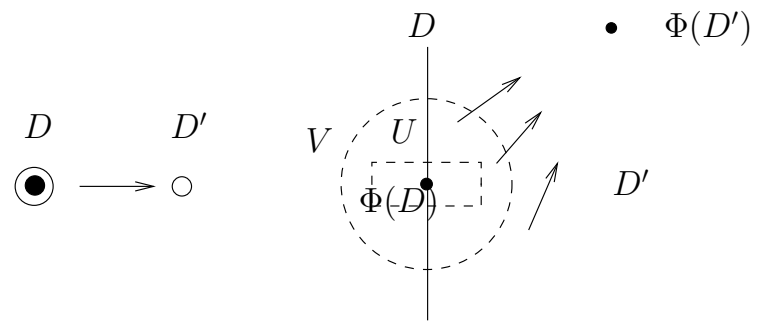

Figure 10: State transition graph and sketch of phase portrait for the proof of Theorem 5.3. Only the transition from $D$ to $D^{\prime}$ is shown.

For the example of Figure 1, the switching domain $D^{7}$ satisfies the criteria of Theorem 5.3 , since there are transitions from $D^{7}$ to the contiguous regulatory domains $D^{1}$ and $D^{13}$. Hence Theorem 5.3 shows that $D^{7}$ contains an unstable singular equilibrium point.

Remark. An analogous theorem can be formulated for $\Pi(D)$.

\subsection{Stronger Conjecture on Stability}

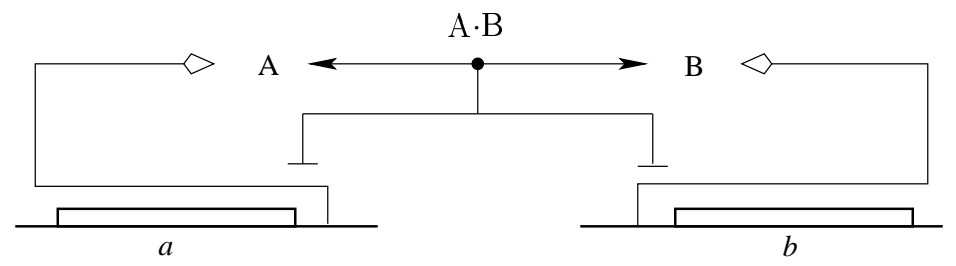

Figure 11: Example of a genetic regulatory network of two genes ( $a$ and $b$ ), each coding for a regulatory protein (A and B). The regulatory proteins form a heterodimer A.B.

The hypotheses of Theorems 5.1 and 5.2, are quite strong and there are many PL models in which we suspect an equilibrium point to be stable even though it is not covered by the criteria of Theorems 5.1 and 5.2. For example, consider the two-gene network illustrated in Figure 11. The proteins A and B form a heterodimer A.B repressing the expression of both genes. The equations modelling the example network can be written as

$$
\begin{aligned}
\dot{x}_{a} & =\kappa_{a}\left(1-s^{+}\left(x_{a}, \theta_{a}\right) s^{+}\left(x_{b}, \theta_{b}\right)\right)-\gamma_{a} x_{a}, \\
\dot{x}_{b} & =\kappa_{b}\left(1-s^{+}\left(x_{a}, \theta_{a}\right) s^{+}\left(x_{b}, \theta_{b}\right)\right)-\gamma_{b} x_{b} .
\end{aligned}
$$

The phase space associated with the model consists of four regulatory domains and five switching domains. The state transition graph for typical parameter values is shown in 


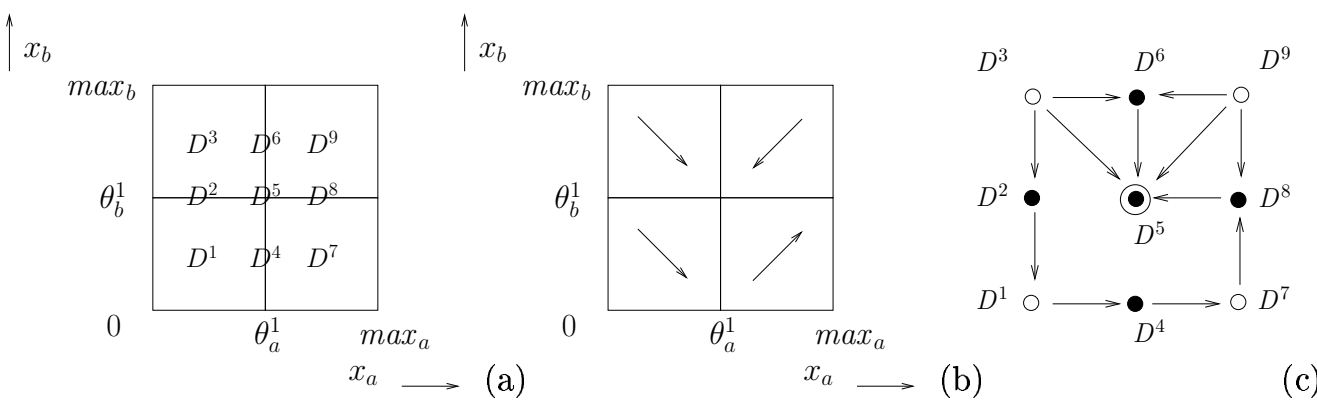

Figure 12: (a) Phase space and (b) vector field for the genetic regulatory network described by the PL model in Equations (30) and (31). (c) State transition graph for the model. The switching domain $D^{5}$ clearly contains a stable equilibrium point, but does not satisfy the hypotheses of Theorems 5.1 and 5.2 since there is no transition from regulatory domains $D^{1}$ and $D^{7}$ to the domain $D^{5}$.

Figure 12. Examination of the phase portrait and the vector field near $D^{5}$ shows that $D^{5}$ clearly contains a stable equilibrium point or set, but does not satisfy the hypotheses of Theorems 5.1 and 5.2 since there is no transition from the regulatory domains $D^{1}$ and $D^{7}$ to the domain $D^{5}$. The following set of neighbouring domains of lower order is useful in formulating Conjecture 5.4. If $D \in \mathcal{D}$ is a domain, define $N(D)$ to be the set of all contiguous domains of $D$ that have $D$ in their boundary.

$$
N(D)=\left\{D^{\prime} \in \mathcal{D} \mid D \subseteq \partial D^{\prime}\right\} .
$$

This set has the property that $\operatorname{order}\left(D^{\prime}\right)<\operatorname{order}(D)$ for all $D^{\prime} \in N(D)$. An improved conjecture with relaxed hypotheses on the state transition graph is stated below. Conjecture 5.4 states that a switching domain $D$ contains an asymptotically stable equilibrium set if the state transition graph contains no outgoing transitions from $D$ and no cycles (see Definition 14) in the neighbouring domains $N(D)$.

Conjecture 5.4. Assume $\Omega \subset \mathbb{R}^{n}$. Let $D \in \mathcal{D}$ be a switching domain containing a singular equilibrium set $\Phi(D)$ that satisfies Assumption 1. Let $G$ be the state transition graph. $\Phi(D)$ is weakly asymptotically stable if

1. G contains no outgoing transitions from $D$, and

2. there is no cycle in the restriction of $G$ to $N(D)$.

Although we do not prove Conjecture 5.4 here, it is clear from Figure 12 that this example satisfies the criteria of the conjecture. Conjecture 5.4 would allow stability of singular equilibria to be proven for a much wider class of PL models of genetic regulatory networks and so a proof of this result is the subject of ongoing work. 


\section{Discussion}

In this paper we have studied the stability of equilibria for piecewise-linear models of genetic regulatory networks. We have focused on the characterization of equilibria on surfaces of discontinuity arising from the use of step functions employed in the PL models. Equilibria that lie on the surfaces of discontinuity (so-called threshold hyperplanes), are referred to as singular equilibria. In order to study these singular equilibria, we use the approach of Filippov to define the solutions on the threshold hyperplanes, leading to a piecewise-linear differential inclusion (PLDI) model. While singular equilibria can sometimes be isolated points, in general they are subsets of the threshold hyperplanes. Our main contributions are to place the stability problem in the context of Filippov solutions, plus the formulation and proof of stability criteria based on a qualitative representation of the dynamics of the system, the state transition graph (STG). Firstly, we feel that using differential inclusions and Filippov solutions clarifies the stability problem for singular equilibria and places it on a firm mathematical footing. Secondly, our results on stability and instability based on the qualitative abstraction of the STG are the most useful in practice and are in keeping with the qualitative nature of the underlying PL models. We also formulated a stronger conjecture on the stability of singular equilibria that we have left unproven.

Assumption 1 is crucial to most of the results presented here. This assumption is that for every domain $D$, either switching or regulatory, the focal set does not intersect with the supporting hyperplane of some domain $D^{\prime}$ that lies in the boundary of $D$. Informally, for regulatory domains this implies that the focal point lies strictly on one side or the other of the threshold hyperplanes in the boundary, thus guaranteeing a transition in finite time. For switching domains it implies that the focal set for $D$ never intersects one of the hyperplanes that support the boundary of $D$, and in particular, if $D$ contains a singular equilibrium, this equilibrium is strictly contained in $D$. Assumption 1 is generic if the focal set is a point (i.e. for regulatory domains or for switching domains in $\mathbb{R}^{2}$ ), in the sense that a specific relationship between the independent parameters $\left\{\theta_{i}^{j}\right\},\left\{\gamma_{i}\right\},\left\{\kappa_{i l}\right\}$ has to be specified for Assumption 1 to be violated. However, in the case when $\Phi(D)$ is a set, Assumption 1 is not generic anymore. If Assumption 1 is violated, then solutions for $D \in \mathcal{D}$ can take infinite time to reach a domain in the boundary of $D$. Additionally, for switching domains, if Assumption 1 is violated then the focal set could span several switching domains, which can sometimes give rise to equilibria that straddle more than one domain. In this case, for every $\phi \in \Phi(D)$, there exist solutions of the PLDI that can transition from one switching domain to another, while remaining inside the equilibrium set itself.

Within the hybrid systems literature, much has been written on the stability of switching and hybrid systems (see, for example the review of Decarlo et al [DBPL00]). One common approach is the use of Multiple Lyapunov Functions to prove Lyapunov stability for switched system [JR98, Bra98]. Many results using Multiple Lyapunov Functions are not directly applicable to systems with sliding modes and/or cases when the domains do not have a common focal point, although they might be extended [Bra98]. However, the structure of the PL system (2) is particular and the problem we consider quite specific, which allows us to take a different approach. Indeed, system (2) has a hyper-rectangular partitioning of 
a high-dimensional phase space, there are powerful results on the monotonic convergence properties of solutions towards the focal sets (Lemma 2.1), and only the singular equilibria, arising from sliding modes on the threshold hyperplanes, are of interest here. These three facts make a more tailored approach possible in this case. However, an application of a generalized Lyapunov function approach to this PL system may be a promising direction for future work and may help prove Conjecture 5.4. Paden and Sastry, and Shevitz and Padden have used Filippov solutions and Clarke's generalized gradient to extend Lyapunov stability results to piecewise smooth systems [PS87, SP94, Cla83], although [SP94] makes assumptions on the uniqueness of solutions that do not hold in the PLDI models we consider here.

The analysis of PL models, in particular equilibria in threshold hyperplanes, has also been the subject of much work in the mathematical biology community. Often, however, the dynamics on the threshold hyperplanes are not defined and the existence of singular equilibria is demonstrated by some other means. For example, Mestl et al [MPO95a], use continuous functions called logoids in the model, then take their limit to approach step functions and approximate the dynamics on the threshold hyperplanes. Similarly, Plahte et al [PMO98] use a more general class of continuous functions in models with switch-like interactions to study the dynamics on the threshold hyperplanes. The work of Snoussi and Thomas [ST93] also does not define the dynamics on the threshold hyperplanes, but infers the existence of singular equilibria based on the behaviour of the vector field around the threshold hyperplanes and by analogy with continuous nonlinear systems. Our approach differs, in that we use Filippov solutions to define the dynamics on the threshold hyperplanes in a mathematically rigorous manner, which is also constructive and practical. Further, Filippov solutions can be used to define singular equilibria that lie on the threshold hyperplanes, to study the stability of such equilibria, and to develop criteria for their stability and instability. The Filippov approach is a standard approach in control theory and has been used in several other problems in mathematical biology. Boukal and Křivan [BK99], for example, define Filippov solutions for predator-prey models with optimal foraging behaviour and demonstrate the existence of a global attractor using a Lyapunov function approach.

An initial aim of future work, from a mathematical perspective, would be to relax Assumption 1, if possible, and extend our approach to study the resulting "weak solutions" that stay within the equilibrium set itself, but can transition from one switching domain to another. Based on the stability criteria proven in Theorems 5.1 and 5.2, we would also like to extend the approach of using Filippov solutions and the state transition graph to develop similar criteria for limit cycles. This would involve extending the work of Glass and Pasternack [GP78] on limit cycles in this class of PL systems. Glass and Pasternack define a state transition graph and study limit cycles, but do not take sliding mode solutions into account. Similarly, a characterization of positively invariant sets could be developed in terms of criteria based on the state transition graph. It would also be interesting to further explore the relations between the dynamical properties of PL systems and those of related asynchronous or synchronous logical models of genetic regulatory networks [Td90, Kau93, DAT ${ }^{+}$03]. 
The qualitative simulation of genetic regulatory networks, leading to the generation of the state transition graph for PL models, has been implemented in the software tool Genetic Network Analyzer (GNA). GNA has been used in collaboration with experimental biologists for the analysis of several genetic regulatory networks of biological interest: the initiation of sporulation in Bacillus subtilis $\left[\mathrm{dJGB}^{+} 04\right.$ ], quorum sensing in Pseudomonas aeruginosa [VF04], and the nutritional stress response in Escherichia coli [RdJP $\left.{ }^{+}\right]$. Based on the work presented in this paper, a module of GNA for identification of all regular and singular equilibria and determination of their stability could be developed. Recent work by Devloo et al, using constraint programming techniques to identify all equilibria in logical models of large genetic regulatory networks, may aid in this [DHL03]. An equilibria and stability module for GNA could be exploited for rapid characterization of attractors and steady state behaviour of large and complex genetic regulatory networks.

\section{Acknowledgments}

The authors would like to thank Tewfik Sari (Laboratoire de Mathématiques, Université de Haute Alsace), Grégory Batt (INRIA Helix) and Frédéric Grognard (INRIA Comore) for their contributions to this paper. The authors acknowledge the financial support of the ARC GDyn initiative at INRIA and the ACI IMPBio BacAttract initiative at the French Ministry for Research.

\section{References}

[ABI $\left.{ }^{+} 01\right]$ R. Alur, C. Belta, F. Ivančíc, V. Kumar, M. Mintz, G.J. Pappas, H. Rubin, and J. Schlug. Hybrid modeling and simulation of biomolecular networks. In M.D. Di Benedetto and A. Sangiovanni-Vincentelli, editors, Hybrid Systems: Computation and Control (HSCC 2001), volume 2034 of LNCS, pages 19-32. Springer-Verlag, 2001.

$\left[\mathrm{BFH}^{+} 04\right]$ C. Belta, P. Finin, L. C. G. J. M. Habets, A. Halasz, M. Imielinksi, V. Kumar, and $H$. Rubin. Understanding the bacterial stringent response using reachability analysis of hybrid systems. In R. Alur and G. Pappas, editors, Hybrid Systems: Computation and Control (HSCC 2004), volume 2993 of LNCS. Springer-Verlag, Berlin, 2004.

[BK99] D. Boukal and V. Křivan. Lyapunov functions for Lotka-Volterra predator-prey models with optimal foraging behavior. J. Math. Biol., 39:493-517, 1999.

[Bra98] M. Branicky. Multiple Lyapunov functions and other analysis tools for switching and hybrid systems. IEEE Trans. Automatic Control, 43(4):175-482, 1998. 
[BS67] N. P. Bhatia and G. P. Szegö. Dynamical Systems: Stability Theory and Applications. Number 35 in Lecture Notes in Mathematics. Springer-Verlag, Berlin, 1967.

[Cla83] F. Clarke. Optimization and Nonsmooth Analysis. Wiley, New York, 1983.

$\left[\mathrm{DAT}^{+} 03\right]$ J. Demongeot, J. Aracena, F. Thuderoz, T. Baum, and O. Cohen. Genetic regulation networks: circuits, regulons and attractors. C.R. Biologies, 326:171$188,2003$.

[DBPL00] R. Decarlo, M. Branicky, S. Pettersson, and B. Lennartson. Perspectives and results on the stability and stabilizability of hybrid systems. Proc. IEEE, 88(7):1069-1083, 2000.

[DHL03] V. Devloo, P. Hansen, and M. Labbé. Identification of all steady states in large networks by logical analysis. Bull. Math. Biol., 65:1025-1051, 2003.

[dJ02] H. de Jong. Modeling and simulation of genetic regulatory systems: A literature review. J. Comput. Biol., 9(1):69-105, 2002.

[dJGB $\left.{ }^{+} 04\right]$ H. de Jong, J. Geiselmann, G. Batt, C. Hernandez, and M. Page. Qualitative simulation of the initiation of sporulation in Bacillus subtilis. Bull. Math. Biol., $6(2): 261-300,2004$.

[dJGH+ $\left.{ }^{+} 4\right]$ H. de Jong, J-L. Gouzé, C. Hernandez, M. Page, T. Sari, and J. Geiselmann. Qualitative simulation of genetic regulatory networks using piecewise-linear models. Bull. Math. Biol., 6(2):301-340, 2004.

[dJGHP03] H. de Jong, J. Geiselmann, C. Hernandez, and M. Page. Genetic Network Analyzer: Qualitative simulation of genetic regulatory networks. Bioinformatics, 19(3):336-344, 2003.

[Edw00] R. Edwards. Analysis of continuous-time switching networks. Physica D, 146:165-199, 2000.

[ES98] C. Edwards and S.K. Spurgeon. Sliding Mode Control: Theory and Applications. Taylor \& Francis, 1998.

[ESAG01] R. Edwards, H.T. Siegelmann, K. Aziza, and L. Glass. Symbolic dynamics and computation in model gene networks. Chaos, 11(1):160-169, 2001.

[Fil88] A. F. Filippov. Differential Equations with Discontinuous Righthand Sides. Kluwer Academic Publishers, Dordrecht, 1988.

[GK73] L. Glass and S. A. Kauffman. The logical analysis of continuous non-linear biochemical control networks. J. Theor. Biol., 39:103-129, 1973. 
[Gla75] L. Glass. Classification of biological networks by their qualitative dynamics. $J$. Theor. Biol., 54(1):85-107, 1975.

[GP78] L. Glass and J.S. Pasternack. Stable oscillations in mathematical models of biological control systems. J. Math. Biol., 6:207-223, 1978.

[GP01] F. Giannakopoulos and K. Pliete. Planar systems of piecewise linear differential equations with a line of discontinuity. Nonlinearity, 14:1611-1632, 2001.

[GS02] J.L. Gouzé and T. Sari. A class of piecewise linear differential equations arising in biological models. Dyn. Syst, 17(4):299-316, 2002.

[GT01] R. Ghosh and C.J. Tomlin. Lateral inhibition through Delta-Notch signaling: A piecewise affine hybrid model. In M.D. Di Benedetto and A. SangiovanniVincentelli, editors, Hybrid Systems: Computation and Control (HSCC 2001), volume 2034 of $L N C S$, pages 232-246. Springer-Verlag, Berlin, 2001.

[HS74] M. W. Hirsch and S. Smale. Differential Equations, Dynamical Systems, and Linear Algebra. Number 60 in Pure and Applied Mathematics. Academic Press, San Diego, 1974.

[JR98] M. Johansson and A. Rantzer. Computation of piecewise quadratic Lyapunov functions for hybrid systems. IEEE Trans. Automatic Control, 43(4):555-559, 1998.

[Kau93] S.A. Kauffman. The Origins of Order: Self-Organization and Selection in Evolution. Oxford University Press, 1993.

[Koh01] K.W. Kohn. Molecular interaction maps as information organizers and simulation guides. Chaos, 11(1):1-14, 2001.

[LN04] R. Leine and H. Nijmeijer. Dynamics and Bifurcations in Non-Smooth Mechanical Systems. Number 18 in Lecture Notes in Applied and Computational Mechanics. Springer-Verlag, Berlin, 2004.

[MPO95a] T. Mestl, E. Plahte, and S.W. Omholt. A mathematical framework for describing and analysing gene regulatory networks. J. Theor. Biol., 176(2):291-300, 1995.

[MPO95b] T. Mestl, E. Plahte, and S.W. Omholt. Periodic solutions in systems of piecewise-linear differential equations. Dyn. Stabil. Syst., 10(2):179-193, 1995.

[PMO94] E. Plahte, T. Mestl, and S.W. Omholt. Global analysis of steady points for systems of differential equations with sigmoid interactions. Dyn. Stabil. Syst., 9(4):275-291, 1994.

$\mathrm{RR} \mathrm{n}^{\circ} 5353$ 
[PMO98] E. Plahte, T. Mestl, and S.W. Omholt. A methodological basis for description and analysis of systems with complex switch-like interactions. J. Math. Biol., 36(4):321-348, 1998.

[PS87] B. Padden and S. S. Sastry. A calculus for computing Filippov's differential inclusion with application to the variable structure control of robot manipulators. IEEE Trans. Circuits Systems, 34:73-82, 1987.

[Pta92] M. Ptashne. A Genetic Switch: Phage $\lambda$ and Higher Organisms. Cell Press \& Blackwell Science, Cambridge, MA, 2nd edition, 1992.

[RdJP $\left.{ }^{+}\right] \quad$ D. Ropers, H. de Jong, M. Page, D. Schneider, and H. Geiselmann. Qualitative simulation of the nutritional stress response in Escherichia coli. In preparation.

[Sno89] E.H. Snoussi. Qualitative dynamics of piecewise-linear differential equations: A discrete mapping approach. Dyn. Stabil. Syst., 4(3-4):189-207, 1989.

[SP94] D. Shevitz and B. Padden. Lyapunov stability theory of nonsmooth systems. IEEE Trans. Automatic Control, 39(9):1910-1914, 1994.

[ST93] E. H. Snoussi and R. Thomas. Logical identification of all steady states: the concept of feedback loop characteristic states. Bull. Math. Biol., 55:973-991, 1993.

[Td90] R. Thomas and R. d'Ari. Biological Feedback. CRC Press, 1990.

[TTK95] R. Thomas, D. Thieffry, and M. Kaufman. Dynamical behaviour of biological regulatory networks: I. Biological role of feedback loops and practical use of the concept of the loop-characteristic state. Bull. Math. Biol., 57(2):247-276, 1995.

[Utk92] V. I. Utkin. Sliding Modes in Control and Optimization. Communications and Control Engineering. Springer-Verlag, Berlin, 1992.

[VF04] A. U. Viretta and M. Fussenegger. Modeling the quorum sensing regulatory network of human-pathogenic Pseudomonas aeruginosa. Biotech. Prog., 20:670$678,2004$.

[YY71] G. Yagil and E. Yagil. On the relation between effector concentration and the rate of induced enzyme synthesis. Biophys. J., 11(1):11-27, 1971. 




\section{Unité de recherche INRIA Sophia Antipolis 2004, route des Lucioles - BP 93 - 06902 Sophia Antipolis Cedex (France)}

Unité de recherche INRIA Futurs : Parc Club Orsay Université - ZAC des Vignes 4, rue Jacques Monod - 91893 ORSAY Cedex (France)

Unité de recherche INRIA Lorraine : LORIA, Technopôle de Nancy-Brabois - Campus scientifique 615, rue du Jardin Botanique - BP 101 - 54602 Villers-lès-Nancy Cedex (France)

Unité de recherche INRIA Rennes : IRISA, Campus universitaire de Beaulieu - 35042 Rennes Cedex (France)

Unité de recherche INRIA Rhône-Alpes : 655, avenue de l'Europe - 38334 Montbonnot Saint-Ismier (France)

Unité de recherche INRIA Rocquencourt : Domaine de Voluceau - Rocquencourt - BP 105 - 78153 Le Chesnay Cedex (France) 